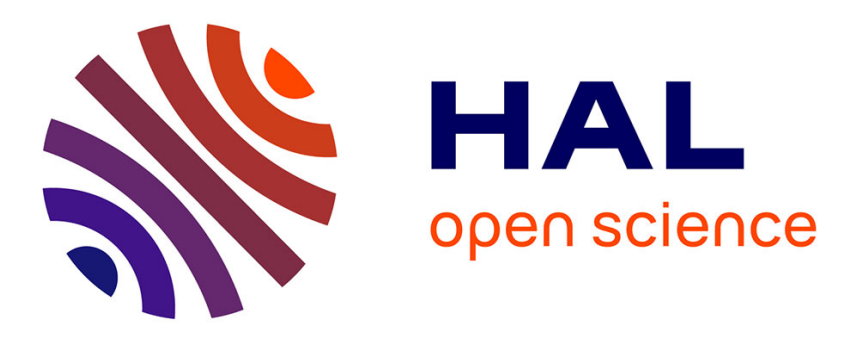

\title{
Multilevel reduced-order computational model in structural dynamics for the low-and medium-frequency ranges
}

Olivier Ezvan, Anas Batou, Christian Soize

\section{- To cite this version:}

Olivier Ezvan, Anas Batou, Christian Soize. Multilevel reduced-order computational model in structural dynamics for the low-and medium-frequency ranges. Computers \& Structures, 2015, 160, pp.111125. 10.1016/j.compstruc.2015.08.007 . hal-01188399

\section{HAL Id: hal-01188399 \\ https://hal.science/hal-01188399}

Submitted on 29 Aug 2015

HAL is a multi-disciplinary open access archive for the deposit and dissemination of scientific research documents, whether they are published or not. The documents may come from teaching and research institutions in France or abroad, or from public or private research centers.
L'archive ouverte pluridisciplinaire HAL, est destinée au dépôt et à la diffusion de documents scientifiques de niveau recherche, publiés ou non, émanant des établissements d'enseignement et de recherche français ou étrangers, des laboratoires publics ou privés. 


\title{
Multilevel reduced-order computational model in structural dynamics for the low- and medium-frequency ranges
}

\author{
O. Ezvan ${ }^{\mathrm{a}}$, A. Batou ${ }^{\mathrm{a}}$ C. Soize ${ }^{* a}$ \\ ${ }^{a}$ Université Paris-Est, Laboratoire Modélisation et Simulation Multi Echelle, MSME UMR 8208 \\ CNRS, 5 bd Descartes, 77454 Marne-la-Vallee, France
}

\begin{abstract}
This work deals with the dynamical analysis of complex structures composed of several structural levels and characterized by the presence of numerous local elastic modes intertwined with global modes, in the medium-frequency range as well as in the low-frequency range. For constructing the ROM, a family of globaldisplacements eigenvectors are calculated and are used instead of the classical elastic modes. Since it is also of importance to adapt the physical models (damping, level of uncertainties, etc) to each one of the structural levels, a multilevel $\mathrm{ROM}$ is proposed. A validation is performed for an automobile complex structure.
\end{abstract}

Keywords: structural dynamics, multilevel reduced-order model, multilevel ROM, computational model, frequency range, medium-frequency range

\section{Introduction}

In linear structural dynamics, the frequency response functions (FRF) present isolated peaks at the resonance frequencies of associated global elastic modes, in the low-frequency (LF) range. In contrast, the high-frequency (HF) range presents rather smooth FRF due to the presence of a high and constant modal density. For complex structures, a medium-frequency (MF) range appears, for which the modal density exhibits large variations over this band $[1,2]$. The use of the first

\footnotetext{
*Corresponding author

Email address: christian. soize@univ-paris-est.fr(C. Soize*)

${ }^{1}$ Phone number 33160957661 / Fax number 33160957799.
} 
eigenmodes (or elastic modes, associated with the first eigenfrequencies) as a projection basis is particularly adapted to construct an accurate reduced-order model (ROM) of small dimension for analyzing the FRF in the low-frequency range $[3,4,5,6,7]$. Statistical energy methods (such as SEA [8]) are generally used for the high-frequency range analysis.

In this work, we are interested in complex structures characterized by the presence of numerous local elastic modes intertwined with global elastic modes, as soon as the low-frequency range. For instance, this unusual feature is related to (1) the presence of flexible parts attached to a stiff master part and (2) to the high complexity of the structure analyzed. For such a case, if the usual modal analysis is used, the ROM that is constructed can be of a very large dimension, due to the unusual presence of the numerous local elastic modes whose contributions are not necessarily significant for prediction of FRFs. This case is typically encountered in the low-frequency vibration of automobiles for which 5 to 10 global elastic modes can be intertwined with about 1,000 local elastic modes in the frequency band $[0,200] \mathrm{Hz}$, or for the dynamics of fuel assemblies in nuclear power plants, which can exhibit about 250 global elastic modes intertwined with about 50, 000 local elastic modes in the frequency band $[0,400] \mathrm{Hz}$.

To circumvent this difficulty, one solution would consist in using a modal sorting method. In general, such an approach is difficult to perform due to the fact that the elastic modes cannot always be defined either as global or as local elastic modes, since they can be combinations of both global and local displacements. Moreover, the contribution of the local displacements become predominant in the elastic modes when the frequency increases, which is such that the global displacements can not easily be detected among the elastic modes.

A second way would consist in using substructuring techniques $[9,10,11$, $12,13,6]$. Such techniques firstly require to develop the computational model in substructures, and secondly that the stiff master part and the flexible parts be well identified. The specification of the work proposed is to develop a methodology that is adapted to a unique computational model without using substructuring data. In addition, for the complex structures we are interested in, it can be difficult to clearly separate the stiff part from the flexible parts.

In this paper, a new multilevel ROM is proposed for analyzing the dynamics of complex structures in the low- and medium-frequency ranges. This work is a 
continuation of previous research $[14,15,16]$. The general strategy proposed in this work relies on the separation of the displacements associated with each structural level (the different levels of stiffness of parts of the structure). The global level is the level associated with the stiff master part, from which the displacements are global. The local level is the level associated with the flexible parts that are attached to the master part, and from which the displacements can be local. In the master part, the global displacements are predominant while the local contributions are often negligible. The new multilevel ROM constructed allows for

(i) obtaining a small-dimension ROM for complex structures using a globaldisplacements basis,

(ii) adapting the physical models (damping, level of uncertainties, etc.) to the different levels of stiffness in the structure, using a unique multilevel ROM based on the use of several vector bases whose displacements correspond to the several distinct levels in the structure.

(i) The first objective of this paper is thus to construct a ROM of smaller dimension than the ROM obtained using classical modal analysis. The strategy used relies on the filtering of the local displacements so as to obtain a ROM whose associated reduction basis may be constituted of global-displacements vectors only. This filtering is performed by solving the usual generalized eigenvalue problem corresponding to the homogeneous conservative system, but in which the kinetic energy is approximated, while the elastic energy is kept exact. The filtering of the local displacements thus relies on the choice of approximation (reduced kinematics) for the kinetic energy. The convergence of the global-displacements ROM constructed is then controlled through the vector subspace associated with the reduced kinematics used.

(ii) The second objective concerns the construction of separated representations adapted to each type of structural level. It is based on the use of the methodology dedicated to the construction of a global-displacements ROM. The choice of vector subspace for the calculation of the kinetic energy allows the displacements associated with each structural level to be either considered as global or as local displacements.

We present a general method for the construction of the global-displacements ROM. The construction of a polynomial approximation for the kinetic energy, adapted to the filtering of the local displacements, is detailed. The formulation of the global-displacements ROM is given, and computational aspects are addressed. 
Based on this method, the construction of the proposed multilevel ROM is presented, using several vector bases whose displacements correspond to the several distinct structural levels. The theoretical part is followed by the presentation of an application devoted to an automobile for which the computational model used has been presented in [15].

\section{Context and strategy}

The reference computational dynamical model is introduced, followed by its classical reduction on elastic modes, allowing both the framework of the present work and the notation used to be introduced. Then, the general strategy proposed for constructing the global-displacements ROM is summarized.

Let $\Omega$ be the bounded domain of a tridimensional linear damped structure that is fixed on a part $\Gamma_{0}$ of its boundary $\partial \Omega$, such that there are no rigid body displacements. The structure is subjected to external loads on the other part $\Gamma$ of $\partial \Omega$. We are interested in predicting the FRFs of the structure in the frequency band of analysis, $\mathcal{B}=\left[\omega_{\min }, \omega_{\max }\right]$, with $0<\omega_{\min }$. The reference computational model is constructed using the finite element method $[17,18]$. For all $\omega$ in $\mathcal{B}$, the complex vector $\mathbb{U}(\omega)$ of the $m$ DOFs of the finite element model, corresponding to the discretization of the displacement field, is the solution of the matrix equation

$$
\left(-\omega^{2}[\mathbb{M}]+i \omega[\mathbb{D}]+[\mathbb{K}]\right) \mathbb{U}(\omega)=\mathbb{F}(\omega),
$$

where $[\mathbb{M}],[\mathbb{D}]$, and $[\mathbb{K}]$ are the $(m \times m)$ positive-definite symmetric real mass, damping, and stiffness matrices. The complex vector $\mathbb{F}(\omega)$ is related to the discretization of the external forces. For the complex dynamical structures of interest, the number $m$ of DOFs can be relatively high (a few millions or a dozen millions).

The first $n$ eigenfrequencies $\left\{f_{\alpha}\right\}_{\alpha}$ and the associated elastic modes $\left\{\boldsymbol{\varphi}_{\alpha}\right\}_{\alpha}$ in $\mathbb{R}^{m}$ are obtained by solving the generalized eigenvalue problem,

$$
[\mathbb{K}] \varphi_{\alpha}=\lambda_{\alpha}[\mathbb{M}] \varphi_{\alpha}
$$

where the positive eigenvalues $\left\{\lambda_{\alpha}\right\}_{\alpha}$ are such that $0<\lambda_{1} \leqslant \ldots \leqslant \lambda_{n}$, from which the eigenfrequencies $\left\{f_{\alpha}\right\}_{\alpha}$ are given by $f_{\alpha}=\sqrt{\lambda_{\alpha}} / 2 \pi$. Let $[\Phi]=\left[\boldsymbol{\varphi}_{1} \ldots \boldsymbol{\varphi}_{n}\right]$ be the $(m \times n)$ real matrix such that $[\Phi]^{T}[\mathbb{K}][\Phi]=[\Lambda]$ and $[\Phi]^{T}[\mathbb{M}][\Phi]=\left[I_{n}\right]$, with $[\Lambda]$ the diagonal matrix of the first $n$ eigenvalues. The classical modal anal- 
ysis method consists in writing, for all $\omega$ in $\mathcal{B}$ and with $n \ll m$,

$$
\mathbb{U}(\omega) \simeq \mathbb{U}^{(n)}(\omega)=\sum_{\alpha=1}^{n} q_{\alpha}(\omega) \boldsymbol{\varphi}_{\alpha}=[\Phi] \mathbf{q}(\omega),
$$

in which the $n$-dimensional complex vector $\mathbf{q}(\omega)$ is a vector of generalized coordinates. The generalized damping matrix $[\mathcal{D}]$ is such that $[\mathcal{D}]=[\Phi]^{T}[\mathbb{D}][\Phi]$. Introducing the generalized force $\mathcal{F}(\omega)=[\Phi]^{T} \mathbb{F}(\omega)$, the classical ROM associated with Eq. (3) is written as

$$
\left(-\omega^{2}\left[I_{n}\right]+i \omega[\mathcal{D}]+[\Lambda]\right) \mathbf{q}(\omega)=\mathcal{F}(\omega) .
$$

For the case of a structure that exhibits numerous local elastic modes, the ROM proposed is constructed by using a basis of a global-displacements space, instead of using all the elastic modes that are present in frequency band $\mathcal{B}$. Let $\mathcal{S}_{\text {glob }}$ be the global-displacements space spanned by some eigenvectors $\left\{\boldsymbol{\psi}_{\alpha}\right\}_{\alpha}$ of the following generalized eigenvalue problem,

$$
[\mathbb{K}] \boldsymbol{\psi}_{\alpha}=\sigma_{\alpha}\left[\mathbb{M}_{\mathcal{A}_{g}}\right] \boldsymbol{\psi}_{\alpha}
$$

corresponding to the homogenous conservative system for which the kinetic energy is approximated while the elastic energy is kept exact. In Eq. (5), $\sigma_{\alpha}$ is the positive eigenvalue associated with $\psi_{\alpha}$ and $\left[\mathbb{M}_{\mathcal{A}_{g}}\right]$ is the modified mass matrix that depends on the approximation subspace $\mathcal{A}_{g}$ associated with a choice of reduced kinematics for the kinetic energy. In previous work [14], the domain of the structure is partitioned into $N_{s}$ subdomains, $\Omega_{1}, \ldots, \Omega_{N_{s}}$, and the reduced kinematics is constructed in choosing the displacement field as a constant in each subdomain. In such a case, only $3 N_{s}$ global eigenvectors, $\left\{\boldsymbol{\psi}_{\alpha}\right\}_{\alpha}$, associated with finite eigenvalues, $\left\{\sigma_{\alpha}\right\}_{\alpha}$, can be obtained because there are only $3 N_{s}$ generalized DOFs constituting the reduced kinematics for the mass matrix ( 3 translations per subdomain). The characteristic dimension of the subdomains allows for controlling the level of filtering of local displacements. In this case, for the continuous formulation, a projection operator $h^{r}$ of the displacement field $\mathbf{u}$ onto the subspace of constant functions by subdomain is introduced, such that, for all $\mathbf{x}$ in $\Omega$,

$$
\left\{h^{r}(\mathbf{u})\right\}(\mathbf{x})=\sum_{j=1}^{N_{s}} \mathbb{1}_{\Omega_{j}}(\mathbf{x}) \frac{1}{m_{j}} \int_{\Omega_{j}} \rho\left(\mathbf{x}^{\prime}\right) \mathbf{u}\left(\mathbf{x}^{\prime}\right) d \mathbf{x}^{\prime},
$$


in which $\mathbb{1}_{\Omega_{j}}(\mathbf{x})=1$ if $\mathbf{x} \in \Omega_{j}$ and is zero otherwise, where $m_{j}=\int_{\Omega_{j}} \rho(\mathbf{x}) d \mathbf{x}$ is the mass of subdomain $\Omega_{j}$, and where $\rho$ is the mass density. The finite element discretization $\left[H^{r}\right]$ of $h^{r}$ is used for obtaining the reduced-kinematics mass matrix $\left[\mathbb{M}_{\mathcal{A}_{g}}\right]=\left[H^{r}\right]^{T}[\mathbb{M}]\left[H^{r}\right]$. In this paper, the theoretical framework is extended to the use of any reduced kinematics for the kinetic energy.

It should be noted that the approximate model for the kinetic energy, represented by matrix $\left[\mathbb{M}_{\mathcal{A}_{g}}\right]$, is introduced for the sole purpose of the construction of subspace $\mathcal{S}_{\text {glob }}$. The ROM will be obtained by projecting the computational model defined by Eq. (1) onto this subspace.

\section{Construction of the reduced-kinematics mass matrix}

In this section, a general framework is provided in order to construct the reduced-kinematics mass matrix $\left[\mathbb{M}_{\mathcal{A}_{g}}\right]$ in Eq. (5). As we have explained, the reduced kinematics defined by $\mathcal{A}_{g}$ is introduced in order to be able to remove the contributions of the local displacements, that is to say in filtering the local displacements.

Let $\mathcal{A}$ denote the $m$-dimensional real vector space equipped with the innerproduct $\left\langle\mathbf{x}, \mathbf{y}>_{\mathbb{M}}=\mathbf{y}^{T}[\mathbb{M}] \mathbf{x}\right.$. Space $\mathcal{A}_{g}$ is a vector subspace of $\mathcal{A}$, for which dimension is denoted $N_{g} \leqslant m$. It should be noted that $N_{g}$ can be chosen greater than $n$. Let $[B]$ be an $\left(m \times N_{g}\right)$ real matrix whose columns constitute an orthonormal vector basis of $\mathcal{A}_{g}$, with respect to the inner-product of $\mathcal{A}$, and which is then such that

$$
[B]^{T}[\mathbb{M}][B]=\left[I_{N_{g}}\right] .
$$

It should be noted that $\mathcal{A}_{g}$ is introduced for constructing matrix $\left[\mathrm{M}_{\mathcal{A}_{g}}\right]$ and consequently, has nothing to do with $\mathcal{S}_{\text {glob }}$. In the following, we present the details concerning the construction of the reduced kinematics for the kinetic energy.

\subsection{Orthogonal-projection matrix}

Any vector $\mathbf{v}$ in $\mathcal{A}_{g}$ can be written as $\mathbf{v}=[B] \mathbf{c}$, in which $\mathbf{c}$ is an $N_{g}$-dimensional real vector of generalized coordinates. For all $\mathbb{V}$ in $\mathcal{A}$, the associated kinetic energy $E_{k}(\mathbb{V})$ is given by $E_{k}(\mathbb{V})=\frac{1}{2} \mathbb{V}^{T}[\mathbb{M}] \mathbb{V}$ and can be written as $E_{k}(\mathbb{V})=$ $\frac{1}{2}\|\mathbb{V}\|_{\mathbb{M}}^{2}$. It should be noted that $E_{k}(\mathbb{V})$ would represent the kinetic energy at time $t$ if $\mathbb{V}$ was the velocity at time $t$. Presently, $\mathbb{V}$ is a real vector independent of $t$ 
and consequently, $E_{k}(\mathbb{V})$ is not a kinetic energy. Nevertheless, in all this paper, the quadratic form $E_{k}$ will be called the kinetic energy by abuse of terminology. For any $\mathbb{V}$ fixed in $\mathcal{A}$, let $\mathbb{V}^{\mathcal{A}_{g}}$ in $\mathbb{R}^{m}$ be the orthogonal projection of $\mathbb{V}$ onto $\mathcal{A}_{g}$. Let $\left[\mathbb{P}_{\mathcal{A}_{g}}\right]$ be the $(m \times m)$ real orthogonal-projection matrix from $\mathcal{A}$ onto $\mathcal{A}_{g}$, of rank $N_{g}$, such that $\mathbb{V}^{\mathcal{A}_{g}}=\left[\mathbb{P}_{\mathcal{A}_{g}}\right] \mathbb{V}$. The $m$-dimensional vector $\mathbb{V}^{\mathcal{A}_{g}}$ can be written as $\mathbb{V}^{\mathcal{A}_{g}}=[B] \mathbf{c}^{\text {opt }}(\mathbb{V})$, in which $\mathbf{c}^{\text {opt }}(\mathbb{V})$ is the unique solution of the optimization problem,

$$
\mathbf{c}^{\mathrm{opt}}(\mathbb{V})=\arg \min _{\mathbf{c} \in \mathbb{R}^{N_{g}}}\|\mathbb{V}-[B] \mathbf{c}\|_{\mathbb{M}}^{2},
$$

and which is given by

$$
\mathbf{c}^{\mathrm{opt}}(\mathbb{V})=\left([B]^{T}[\mathbb{M}][B]\right)^{-1}[B]^{T}[\mathbb{M}] \mathbb{V} .
$$

Using Eq. (7), matrix $\left[\mathbb{P}_{\mathcal{A}_{g}}\right]$ is written as

$$
\left[\mathbb{P}_{\mathcal{A}_{g}}\right]=[B][B]^{T}[\mathbb{M}] .
$$

It can be seen that the construction proposed is such that the residual kinetic energy $E_{k}(\mathbb{V})-E_{k}\left(\mathbb{V}^{\mathcal{A}_{g}}\right)=E_{k}\left(\mathbb{V}-\mathbb{V}^{\mathcal{A}_{g}}\right)$ is minimum.

\subsection{Reduced-kinematics mass matrix}

Definition. For all $\mathbb{V}$ in $\mathcal{A}$, we have $\mathbb{V}^{\mathcal{A}_{g}}=\left[\mathbb{P}_{\mathcal{A}_{g}}\right] \mathbb{V}$, for which the associated kinetic energy $E_{k}\left(\mathbb{V}^{\mathcal{A}_{g}}\right)$ is thus given by $E_{k}\left(\mathbb{V}^{\mathcal{A}_{g}}\right)=\frac{1}{2} \mathbb{V}^{\mathcal{A}_{g}}[\mathbb{M}] \mathbb{V}^{\mathcal{A}_{g}}$, which can be rewritten as

$$
E_{k}\left(\mathbb{V}^{\mathcal{A}_{g}}\right)=\frac{1}{2} \mathbb{V}^{T}\left[\mathbb{M}_{\mathcal{A}_{g}}\right] \mathbb{V}
$$

in which $\left[\mathbb{M}_{\mathcal{A}_{g}}\right]$ is the mass matrix, which is constructed with the kinematics defined by space $\mathcal{A}_{g}$, and which is written as

$$
\left[\mathbb{M}_{\mathcal{A}_{g}}\right]=\left[\mathbb{P}_{\mathcal{A}_{g}}\right]^{T}[\mathbb{M}]\left[\mathbb{P}_{\mathcal{A}_{g}}\right]
$$

Positiveness and null space. Since matrix $[\mathbb{M}]$ is positive definite, from Eq. (12) it can be deduced that the null space of $\left[\mathbb{M}_{\mathcal{A}_{g}}\right]$ is equal to the null space of $\left[\mathbb{P}_{\mathcal{A}_{g}}\right]$, which is not reduced to $\{0\}$, and that matrix $\left[\mathbb{M}_{\mathcal{A}_{g}}\right]$ is positive semidefinite.

Mass conservation. Let $m^{\text {tot }}$ be the positive number such that $m^{\text {tot }}=\frac{1}{3} \mathbf{1}^{T}[\mathbb{M}] \mathbf{1}$, in which the $m$-dimensional vector $\mathbf{1}$ is constituted of ones for the translation 
DOFs and of zeros for the rotation DOFs (if there exist in the computational model). Quantity $m^{\text {tot }}$ represents approximately the mass of the structure (the mass located on the fixed boundary conditions is not taken into account). If 1 belongs to $\mathcal{A}_{g}$ (thus $\left[\mathbb{P}_{\mathcal{A}_{g}}\right] \mathbf{1}=\mathbf{1}$ ), then the mass $m_{\mathcal{A}_{g}}^{\text {tot }}=\frac{1}{3} \mathbf{1}^{T}\left[\mathbb{M}_{\mathcal{A}_{g}}\right] \mathbf{1}$ is such that $m_{\mathcal{A}_{g}}^{\text {tot }}=m^{\text {tot }}$, which means that the mass is conserved in the kinematics reduction.

Matrix construction. Using Eqs. (7) and (10), Eq. (12) yields

$$
\left[\mathbb{M}_{\mathcal{A}_{g}}\right]=[\mathbb{M}][B][B]^{T}[\mathbb{M}]
$$

It should be noted that matrix $\left[\mathbb{M}_{\mathcal{A}_{g}}\right]$ is generally not sparse and consequently, requires a high memory storage (which is also the case for matrix $\left[\mathbb{P}_{\mathcal{A}_{g}}\right]$ ). Nevertheless, the explicit construction and storage of these matrices will not be necessary.

\subsection{Construction of the reduced kinematics}

The objective of this section is the construction of basis matrix $[B]$ of $\mathcal{A}_{g}$, subspace in which any vector $\mathbf{v}$ can be written as $\mathbf{v}=[B] \mathbf{c}$. In the work initialized in [14] and applied in $[15,16]$, the construction of the reduced kinematics is based on a partitioning of domain $\Omega$ into $N_{s}$ subdomains $\Omega_{1}, \ldots, \Omega_{N_{s}}$. For complex finite element models, such domain partitioning is not a straightforward task. In $[15,16]$, homogeneous partitionings of the finite element mesh of automobiles were performed using an algorithm [15] based on the Fast Marching Method [19, 20]. Nevertheless, the use of more accurate approximations for the kinematics reduction allows for avoiding such a domain partitioning.

In this work, we propose of polynomial approximation of degree $N_{d}$ over the entire domain $\Omega$ of the structure. To do so, $N_{\mu}$ multivariate orthogonal polynomials $\left\{p_{\boldsymbol{\alpha}^{(\mu)}}\right\}_{\boldsymbol{\alpha}^{(\mu)}}$ are used, where for $\mu=1, \ldots, N_{\mu}$ multi-index $\boldsymbol{\alpha}^{(\mu)}$ belongs to some set $\mathcal{K}_{N_{d}}$ that will be defined after. Denoting as $N_{f}$ the number of free nodes of the finite element model, the approximate displacement $v_{\nu}^{d}$ of node $\nu \in\left\{1, \ldots, N_{f}\right\}$ following direction $d$ is written as

$$
v_{\nu}^{d}=\sum_{\boldsymbol{\alpha}^{(\mu)} \in \mathcal{K}_{N_{d}}} p_{\boldsymbol{\alpha}^{(\mu)}}\left(\mathbf{X}_{\nu}\right) c_{\mu}^{d},
$$

in which $\left\{c_{\mu}^{d}\right\}_{\mu}$ are the polynomials coefficients and where $\mathbf{X}_{\nu}=\left(x_{\nu}, y_{\nu}, z_{\nu}\right)$ is the position vector of node $\nu$ at which the polynomials are evaluated. In matrix form, 
the $N_{f}$ equations associated with Eq. (14) can be rewritten as

$$
\mathbf{v}^{d}=[\mathcal{P}] \mathbf{c}^{d},
$$

in which $\mathbf{v}^{d}$ is the $N_{f}$-dimensional real vector of the approximate displacements $\left\{v_{\nu}^{d}\right\}_{\nu}$ following direction $d$ and where $\mathbf{c}^{d}$ is the $N_{\mu}$-dimensional real vector of coefficients $\left\{c_{\mu}^{d}\right\}_{\mu}$. The $\left(N_{f} \times N_{\mu}\right)$ real matrix [P ] is constituted of the $N_{f}$ values of each of the $N_{\mu}$ polynomials $\left\{p_{\boldsymbol{\alpha}^{(\mu)}}\right\}_{\boldsymbol{\alpha}^{(\mu)}}$ at the mesh nodes. Basis matrix [B] of subspace $\mathcal{A}_{g}$, which is such that $\mathbf{v}=[B] \mathbf{c}$, is then assembled using $[\mathcal{P}]$. It should be noted that only the 3 translational directions are considered for constructing the reduced kinematics of the displacements, which is used for constructing the approximation of the kinetic energy. We thus have $N_{g}=3 N_{\mu}$. This approximation of the kinetic energy is only used for constructing the reduced-order basis that exhibits both translation and rotation DOFs. Therefore, the global-displacements ROM is obtained by projection of the reference computational model using this basis, and consequently, the translations and the rotations are kept in this projection, in particular for the mass matrix.

Let $\mathcal{K}_{N_{d}}$ be the set of the $N_{\mu}$ vectors $\boldsymbol{\alpha}=\left(\alpha_{1}, \alpha_{2}, \alpha_{3}\right)$ such that, for fixed $\alpha_{1}$ in $\left\{0, \ldots, N_{d}\right\}, \alpha_{2}$ belongs to $\left\{0, \ldots, \alpha_{1}\right\}$, and for so given $\alpha_{1}$ and $\alpha_{2}, \alpha_{3}$ belongs to $\left\{0, \ldots, \alpha_{2}\right\}$. It can be deduced that $N_{\mu}=\left(N_{d}+1\right)\left(N_{d}+2\right)\left(N_{d}+3\right) / 6$. Using the same notation as for $\left\{p_{\boldsymbol{\alpha}^{(\mu)}}\right\}_{\boldsymbol{\alpha}^{(\mu)}}$, the values of the multivariate monomials $\left\{m_{\boldsymbol{\alpha}^{(\mu)}}\right\}_{\boldsymbol{\alpha}^{(\mu)}}$ at the mesh nodes can be written as

$$
m_{\boldsymbol{\alpha}^{(\mu)}}\left(\mathbf{X}_{\nu}\right)=x_{\nu}^{\alpha_{1}-\alpha_{2}} y_{\nu}^{\alpha_{2}-\alpha_{3}} z_{\nu}^{\alpha_{3}} .
$$

Similarly to $[\mathcal{P}]$, let $[\mathcal{M}]$ be the $\left(N_{f} \times N_{\mu}\right)$ real matrix that is constituted of the $N_{f}$ values of each of the $N_{\mu}$ monomials $\left\{m_{\boldsymbol{\alpha}^{(\mu)}}\right\}_{\boldsymbol{\alpha}(\mu)}$ at the mesh nodes. Then, matrix $[\mathcal{P}]$ is constructed as an orthonormalization of $[\mathcal{M}]$. To do so, the $\mathrm{QR}$ algorithm is used. In order to satisfy Eq. (7), the orthonormalization is performed with respect to the inner-product of $\mathcal{A}$.

Remark. The proposed reduced kinematics, which is presently applied to whole domain $\Omega$, can also be applied for each subdomain $\Omega_{1}, \ldots, \Omega_{N_{s}}$ of a partition of $\Omega$. If such a partition is introduced, and if the degree $N_{d}$ of the polynomial approximation is chosen, for each subdomain, as

(i) $N_{d}=0$ (which corresponds to a constant displacement field by subdomain), we then obtain the formulation introduced in [14] and in such a case, the projector is defined by Eq. (6) for the continuous case. 
(ii) $N_{d}=1$, then the reduced kinematics is very close to a rigid body displacements field by subdomain as proposed in [23].

\section{Global-displacements ROM}

\subsection{Effective construction of the ROM}

We are interested in constructing a ROM for predicting the global displacements of the stiff master part and not the local displacements of the attached flexible parts. In this method, the filtering of the local displacements is performed using the approximation previously proposed for the mass matrix.

Let $0<\sigma_{1} \leqslant \ldots \leqslant \sigma_{n_{g}}$ be the first $n_{g}$ positive eigenvalues of the generalized eigenvalue problem defined by Eq. (5). Let $[\Psi]=\left[\boldsymbol{\psi}_{1} \ldots \boldsymbol{\psi}_{n_{g}}\right]$ be the $\left(m \times n_{g}\right)$ real matrix of the associated eigenvectors $\left\{\boldsymbol{\psi}_{\alpha}\right\}_{\alpha}$. Dimension $n_{g}$ must be chosen as $n_{g} \leqslant N_{g}$. It should be noted that if $n_{g}$ was chosen greater than $N_{g}$, then the eigenvalues $\sigma_{N_{g}+1} \leqslant \ldots \leqslant \sigma_{n_{g}}$ would be infinite, because the rank of $\left[\mathbb{M}_{\mathcal{A}_{g}}\right]$ is $N_{g}$. Let $\mathcal{S}_{g l o b}$ be the subspace of $\mathbb{R}^{m}$ spanned by $\left\{\boldsymbol{\psi}_{1} \ldots \boldsymbol{\psi}_{n_{g}}\right\}$ and let $\mathcal{S}_{\text {glob }}^{c}$ be its complexified space. Introducing the approximation $\mathbb{U}^{\left(N_{d}, n_{g}\right)}$ of $\mathbb{U}$ such that

$$
\mathbb{U}^{\left(N_{d}, n_{g}\right)}(\omega)=[\Psi] \mathbf{r}(\omega),
$$

the associated ROM is obtained by projecting Eq. (1) onto $\mathcal{S}_{\text {glob }}^{c}$, which yields

$$
\left(-\omega^{2}\left[M^{g}\right]+i \omega\left[D^{g}\right]+\left[K^{g}\right]\right) \mathbf{r}(\omega)=\mathbf{f}^{g}(\omega),
$$

in which $\mathbf{r}(\omega)$ is an $n_{g}$-dimensional complex vector of generalized coordinates and where, for $(A, \mathbb{A}) \in\{(M, \mathbb{M}),(D, \mathbb{D}),(K, \mathbb{K})\}$, we have $\left[A^{g}\right]=[\Psi]^{T}[\mathbb{A}][\Psi]$, and with $\mathbf{f}^{g}(\omega)=[\Psi]^{T} \mathbb{F}(\omega)$. The homogeneous conservative system associated with Eq. (18) yields the small-dimension generalized eigenvalue problem

$$
\left[K^{g}\right] \mathbf{r}_{\alpha}=\lambda_{\alpha}^{g}\left[M^{g}\right] \mathbf{r}_{\alpha},
$$

whose positive eigenvalues $0<\lambda_{1}^{g} \leqslant \ldots \leqslant \lambda_{n_{g}}^{g}$ yield the eigenfrequencies $f_{\alpha}^{g}=$ $\sqrt{\lambda_{\alpha}^{g}} / 2 \pi$. Let $[R]=\left[\mathbf{r}_{1} \ldots \mathbf{r}_{n_{g}}\right]$ be the $\left(n_{g} \times n_{g}\right)$ real matrix of the corresponding eigenvectors, which is normalized such that $[R]^{T}\left[M^{g}\right][R]=\left[I_{n_{g}}\right]$. In Eq. (18), vector $\mathbf{r}(\omega)$ is written as $\mathbf{r}(\omega)=[R] \mathbf{q}^{g}(\omega)$, in which $\mathbf{q}^{g}(\omega)$ is an $n_{g}$-dimensional complex vector of generalized coordinates. For all $\omega$ in frequency band $\mathcal{B}$, the 
global-displacements ROM is then written as

$$
\begin{array}{r}
\mathbb{U}(\omega) \simeq \mathbb{U}^{\left(N_{d}, n_{g}\right)}(\omega)=\left[\Phi^{g}\right] \mathbf{q}^{g}(\omega), \\
\left(-\omega^{2}\left[I_{n_{g}}\right]+i \omega\left[\mathcal{D}^{g}\right]+\left[\Lambda^{g}\right]\right) \mathbf{q}^{g}(\omega)=\mathcal{F}^{g}(\omega),
\end{array}
$$

in which $\left[\Phi^{g}\right]=[\Psi][R]=\left[\varphi_{1}^{g} \ldots \varphi_{n_{g}}^{g}\right]$ is the $\left(m \times n_{g}\right)$ real matrix of the $n_{g}$ global modes $\left\{\boldsymbol{\varphi}_{\alpha}^{g}\right\}_{\alpha}$, with $\left[\Lambda^{g}\right]=\left[\Phi^{g}\right]^{T}[\mathbb{K}]\left[\Phi^{g}\right]$ and $\left[\mathcal{D}^{g}\right]=\left[\Phi^{g}\right]^{T}[\mathbb{D}]\left[\Phi^{g}\right]$. The $n_{g}$ global modes constitute an orthogonal vector basis of $\mathcal{S}_{\text {glob }}$ for the innerproduct $<\mathbf{x}, \mathbf{y}>_{\mathbb{K}}=\mathbf{y}^{T}[\mathbb{K}] \mathbf{x}$.

\subsection{Convergence analysis and computational aspects}

In Eq. (20), the modal contributions of the local modes are not taken into account. The convergence analysis of $\mathbb{U}^{\left(N_{d}, n_{g}\right)}(\omega)$ towards $\mathbb{U}(\omega)$ in frequency band $\mathcal{B}$ must be carried out with respect to degree $N_{d}$ and, for fixed $N_{d}$, with respect to dimension $n_{g}$. In comparison to the usual modal analysis method, it should be noted that the method converges towards the results given by the usual modal analysis when $N_{d}$ increases (see, for instance, Fig. 7). In addition, if $\mathcal{A}_{g}=\mathcal{A}$, then the proposed theory coincides with the usual modal analysis. An example of convergence analysis is given in Section 6.1.

Although matrix $\left[\mathbb{M}_{\mathcal{A}_{g}}\right]$ is a full $(m \times m)$ real matrix, its assembly can be avoided by using an iterative eigensolver (such as the subspace iteration method $[3,24])$. Nevertheless, in the context of the use of commercial softwares, the extraction of the stiffness matrix $[\mathbb{K}]$ can be difficult or even impossible in the case of a black-box software. We thus propose a method (called indirect method) for computing the global-displacements basis represented by matrix $[\Psi]$, which consists in working into the subspace $\mathcal{S}_{\text {elas }}$ that is spanned by the first $n$ elastic modes $\left\{\boldsymbol{\varphi}_{\alpha}\right\}_{\alpha}$. This method allows for avoiding both the assembly of $\left[\mathbb{M}_{\mathcal{A}_{g}}\right]$ and the extraction of $[\mathbb{K}]$. Concerning the computational complexity, the overall cost is dominated by the computation of the $n$ usual elastic modes. For constructing the global-displacements ROM, the additional cost is negligible. Once this ROM is constructed, the gain is generally important because its dimension is smaller than the dimension of the ROM that would be required with the usual modal analysis (due to the presence of numerous local elastic modes). It should be noted that the convergence analysis of the ROM with respect to $N_{d}$ is also carried out with a reduced cost, since, as will be explained after, it involves solving eigenvalue problems that are of reduced dimension. Global eigenvectors $\left\{\boldsymbol{\psi}_{\alpha}\right\}_{\alpha}$ are written 


$$
\boldsymbol{\psi}_{\alpha}=[\Phi] \mathbf{s}_{\alpha}
$$

where the $n$-dimensional real vectors $\left\{\mathbf{s}_{\alpha}\right\}_{\alpha}$ are generalized coordinates, which are the solutions of the reduced-order generalized eigenvalue problem

$$
[\Lambda] \mathbf{s}_{\alpha}=\sigma_{\alpha}\left[M_{\mathcal{A}_{g}}\right] \mathbf{s}_{\alpha} \text {. }
$$

The $(n \times n)$ real mass matrix $\left[M_{\mathcal{A}_{g}}\right]$ is a positive-semidefinite symmetric matrix of rank $\mathcal{N}_{g}$, such that $\mathcal{N}_{g} \leqslant N_{g}$ and $\mathcal{N}_{g} \leqslant n$, and is defined by

$$
\left[M_{\mathcal{A}_{g}}\right]=[\Phi]^{T}\left[\mathbb{M}_{\mathcal{A}_{g}}\right][\Phi] .
$$

Let $[S]=\left[\mathbf{s}_{1} \ldots \mathbf{s}_{n_{g}}\right]$ be the matrix of the eigenvectors associated with the first $n_{g} \leqslant \mathcal{N}_{g}$ finite positive eigenvalues $0<\sigma_{1} \leqslant \ldots \leqslant \sigma_{n_{g}}$. Accordingly to Eq. (22), we have $[\Psi]=[\Phi][S]$. Similarly to the direct approach presented before, global modes $\left\{\varphi_{\alpha}^{g}\right\}_{\alpha}$ associated with the eigenfrequencies $\left\{f_{\alpha}^{g}\right\}_{\alpha}$ are written as $\varphi_{\alpha}^{g}=$ $[\Psi] \mathbf{r}_{\alpha}$, in which $\left\{\mathbf{r}_{\alpha}\right\}_{\alpha}$ are the solutions of the eigenvalue problem defined by Eq. (19), for which the matrices $\left[K^{g}\right]$ and $\left[M^{g}\right]$ are rewritten as

$$
\begin{aligned}
{\left[K^{g}\right] } & =[\Sigma], \\
{\left[M^{g}\right] } & =[S]^{T}[S],
\end{aligned}
$$

in which $[\Sigma]$ is the diagonal matrix of the first $n_{g}$ eigenvalues $\left\{\sigma_{\alpha}\right\}_{\alpha}$. Concerning the construction of matrix $\left[M_{\mathcal{A}_{g}}\right]$, it can be rewritten as

$$
\left[M_{\mathcal{A}_{g}}\right]=[Z][Z]^{T},
$$

in which the $\left(n \times N_{g}\right)$ real matrix $[Z]$ is given by

$$
[Z]=[\Phi]^{T}[\mathbb{M}][B] \text {. }
$$

For each degree $N_{d}$ used in the convergence analysis, matrix $\left[M_{\mathcal{A}_{g}}\right]$ has to be constructed. Depending on $N_{g}$ (which rapidly increases with respect to $N_{d}$ ), i) the matrix product between $[\Phi]^{T}[\mathrm{M}]$ and $[B]$ as well as ii) the QR decomposition for the construction of $[B]$ can be computationally quite demanding. As the increase of $N_{d}$ only amounts to adding higher degree polynomials, we propose that the calculations mentioned in i) and ii) hereinbefore are done once, this for the largest 
degree $N_{d}$. This way, matrix $[Z]$ that is associated with a lower degree $N_{d}$ can be obtained by extracting the corresponding first columns.

\section{Multilevel ROM}

In this section, a methodology is presented for constructing a multilevel ROM, using several vector bases whose displacements correspond to the several distinct structural levels. This methodology is based on the developments presented before, using several levels of filtering of the local displacements. The strategy consists in splitting the admissible displacements space into a direct sum of subspaces. Each subspace is spanned by a family of modes whose displacements are associated with a given structural level.

In general, the local displacements are associated with vibrations having a more or less small wavelength, as encountered in the MF and HF bands. However, the paper concerns complex structures for which local displacements also appear in the LF range. Furthermore, the MF and/or the HF ranges are often studied using adapted physical models to each frequency band. For instance, it is well known that the small-wavelength local elastic modes are very sensitive to uncertainties, while the global elastic modes are relatively more robust with respect to uncertainties. For the complex structures that are considered in this paper, it is assumed that there is a mixing of the typical LF and HF behaviors in the LF range, due to the intertwining of the local displacements with the global displacements in the LF range, which also occurs in the MF range [1]. It is thus beneficial of being able, not only to extract the global displacements from the local displacements for the construction of a small-dimension ROM, but also and more generally, to separate all the scales of displacements (associated with each structural level). The theory for constructing the multilevel ROM will be exposed considering three scales of displacements denoted as $\mathcal{L}, \mathcal{M}$, and $\mathcal{H}$.

- For the first scale, $\mathcal{L}$, the displacements are associated with global displacements (as in LF) belonging to a subspace denoted by $\mathcal{S}_{\mathcal{L}}$.

- For the second scale, $\mathcal{M}$, the displacements are associated with local displacements (as in MF) belonging to a subspace denoted by $\mathcal{S}_{\mathcal{M}}$. These displacements constitute the first scale of the local displacements.

- For the third scale, $\mathcal{H}$, the displacements are associated with local displacements (as in HF) belonging to a subspace denoted by $\mathcal{S}_{\mathcal{H}}$. These displacements constitute the second scale of the local displacements. 


\subsection{Formulation of a multilevel ROM}

The formulation of the multilevel ROM proposed is partly based on the projection of the computational model onto subspace $\mathcal{S}_{\text {glob }}$ whose construction has been presented in Section 4. This first filtering of local displacements allows for obtaining a small-dimension representation for the displacements (belonging to $\mathcal{S}_{\text {glob }}$ and given by Eqs (20)) with a controlled accuracy. The second step of the proposed formulation consists in using several levels of filtering of the local displacements, in order to construct the LF-, MF-, and HF-scale modal bases (spanning subspaces $\mathcal{S}_{\mathcal{L}}, \mathcal{S}_{\mathcal{M}}$, and $\mathcal{S}_{\mathcal{H}}$ of $\mathcal{S}_{\text {glob }}$ ). Since polynomial degree $N_{d}$ can be chosen arbitrarily (or even $\mathcal{A}_{g}$ set as $\mathcal{A}_{g}=\mathcal{A}$ ), the global-displacements $\mathrm{ROM}$ is actually constructed upon the use of more or less local displacements. Let $\mathcal{A}_{\mathcal{L}}$ be the vector subspace whose associated reduced kinematics is adapted for representing the LF scale only and consequently, which contains very few local displacements. Similarly, let $\mathcal{A}_{\mathcal{M}}$ be the vector subspace whose associated reduced kinematics is adapted for representing the LF and MF scales only. Figure 1 illustrates the strategy, which consists in splitting subspace $\mathcal{S}_{\text {glob }}$ (obtained using the reduced kinematics associated with $\mathcal{A}_{g}$ ) into subspaces $\mathcal{S}_{\mathcal{L}}, \mathcal{S}_{\mathcal{M}}$, and $\mathcal{S}_{\mathcal{H}}$, which are non-overlapping, and which are obtained by means of two intermediate levels of filtering associated with $\mathcal{A}_{\mathcal{L}}$ and $\mathcal{A}_{\mathcal{M}}$. We now present an algebraic construction of vector bases associated with these subspaces.
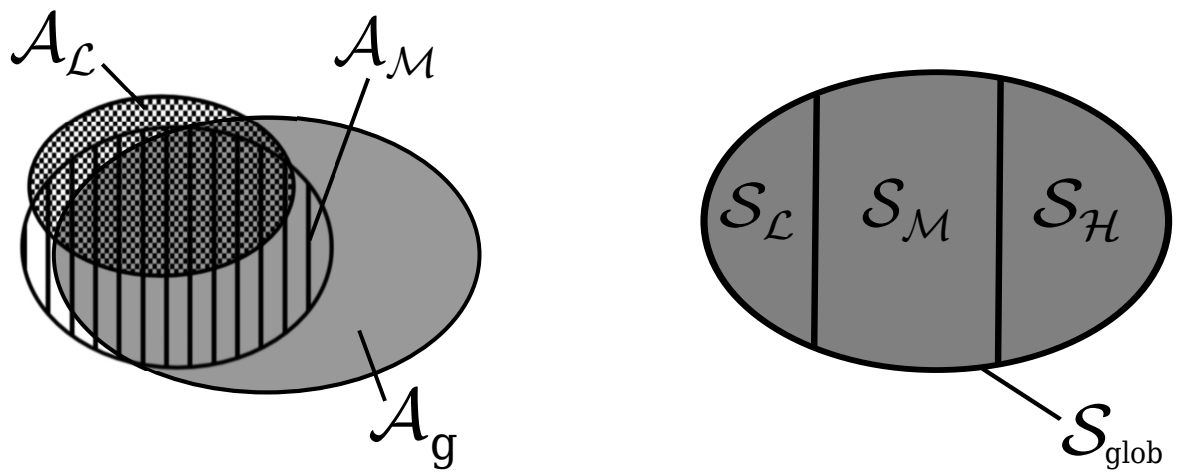

Figure 1: Scheme showing the overlap of approximation subspaces $\mathcal{A}_{g}, \mathcal{A}_{\mathcal{L}}$, and $\mathcal{A}_{\mathcal{M}}$ (left) and scheme representing subspace $\mathcal{S}_{\text {glob }}=\mathcal{S}_{\mathcal{L}} \cup \mathcal{S}_{\mathcal{M}} \cup \mathcal{S}_{\mathcal{H}}$ such that $\mathcal{S}_{\mathcal{L}} \cap \mathcal{S}_{\mathcal{M}} \cap \mathcal{S}_{\mathcal{H}}=\varnothing$ (right).

\subsubsection{LF-scale basis}

We work within a subspace $\mathcal{S}_{\text {glob }}$ of dimension $n_{g}$, associated with a globaldisplacements ROM that has been constructed as presented in Section 4 and whose accuracy is adjusted to the required level through a convergence analysis with 
respect to parameters $N_{d}$ and $n_{g}$. This ROM is represented by matrix $\left[\Lambda^{g}\right]$ of eigenvalues and by reduction basis $\left[\Phi^{g}\right]$. The LF-scale eigenvectors $\left\{\boldsymbol{\psi}_{\alpha}^{\mathcal{L}}\right\}_{\alpha}$ are represented in $\mathcal{S}_{\text {glob }}$ by

$$
\psi_{\alpha}^{\mathcal{L}}=\left[\Phi^{g}\right] \mathbf{s}_{\alpha}^{\mathcal{L}},
$$

in which the $n_{g}$-dimensional real vectors $\left\{\mathbf{s}_{\alpha}^{\mathcal{L}}\right\}_{\alpha}$ are generalized coordinates, which are the solutions of the small-dimension generalized eigenvalue problem

$$
\left[\Lambda^{g}\right] \mathbf{s}_{\alpha}^{\mathcal{L}}=\sigma_{\alpha}^{\mathcal{L}}\left[M_{\mathcal{A}_{\mathcal{L}}}\right] \mathbf{s}_{\alpha}^{\mathcal{L}},
$$

in which the $\left(n_{g} \times n_{g}\right)$ real mass matrix $\left[M_{\mathcal{A}_{\mathcal{L}}}\right]$ is a positive-semidefinite symmetric matrix which is written as

$$
\left[M_{\mathcal{A}_{\mathcal{L}}}\right]=\left[\Phi^{g}\right]^{T}\left[\mathbb{M}_{\mathcal{A}_{\mathcal{L}}}\right]\left[\Phi^{g}\right]
$$

The rank $\mathcal{N}_{\mathcal{L}}$ of matrix $\left[M_{\mathcal{A}_{\mathcal{L}}}\right]$ verifies the inequalities $\mathcal{N}_{\mathcal{L}} \leqslant N_{\mathcal{L}}$ and $\mathcal{N}_{\mathcal{L}} \leqslant$ $n_{g}$ in which $N_{\mathcal{L}}$ is the rank of matrix $\left[\mathbb{M}_{\mathcal{A}_{\mathcal{L}}}\right]$. Let $\left[S^{\mathcal{L}}\right]=\left[\mathbf{s}_{1}^{\mathcal{L}} \ldots \mathbf{s}_{n_{\mathcal{L}}}^{\mathcal{L}}\right]$ be the $\left(n_{g} \times n_{\mathcal{L}}\right)$ real matrix of the eigenvectors associated with the first $n_{\mathcal{L}}$ finite positive eigenvalues $0<\sigma_{1}^{\mathcal{L}} \leqslant \ldots \leqslant \sigma_{n_{\mathcal{L}}}^{\mathcal{L}}$. The matrix of the LF-scale eigenvectors $\left\{\boldsymbol{\psi}_{\alpha}^{\mathcal{L}}\right\}_{\alpha}$ is written as $\left[\Psi^{\mathcal{L}}\right]=\left[\Phi^{g}\right]\left[S^{\mathcal{L}}\right]$. The $n_{\mathcal{L}}$ LF-scale modes $\left\{\boldsymbol{\varphi}_{\alpha}^{\mathcal{L}}\right\}_{\alpha}$ are then defined by

$$
\varphi_{\alpha}^{\mathcal{L}}=\left[\Psi^{\mathcal{L}}\right] \mathbf{r}_{\alpha}^{\mathcal{L}}
$$

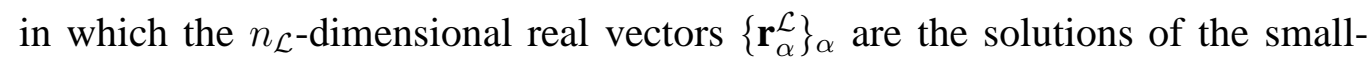
dimension generalized eigenvalue problem

$$
\left[K^{\mathcal{L}}\right] \mathbf{r}_{\alpha}^{\mathcal{L}}=\lambda_{\alpha}^{\mathcal{L}}\left[M^{\mathcal{L}}\right] \mathbf{r}_{\alpha}^{\mathcal{L}}
$$

in which $\left[K^{\mathcal{L}}\right]$ and $\left[M^{\mathcal{L}}\right]$ are positive-definite symmetric $\left(n_{\mathcal{L}} \times n_{\mathcal{L}}\right)$ real matrices that are such that $\left[K^{\mathcal{L}}\right]=\left[\Sigma^{\mathcal{L}}\right]$ and $\left[M^{\mathcal{L}}\right]=\left[S^{\mathcal{L}}\right]^{T}\left[S^{\mathcal{L}}\right]$, with $\left[\Sigma^{\mathcal{L}}\right]$ the diagonal matrix of the first $n_{\mathcal{L}}$ eigenvalues $\left\{\sigma_{\alpha}^{\mathcal{L}}\right\}_{\alpha}$. The generalized eigenvalue problem defined by Eq. (33) admits $n_{\mathcal{L}}$ real eigenvalues $0<\lambda_{1}^{\mathcal{L}} \leqslant \ldots \leqslant \lambda_{n_{\mathcal{L}}}^{\mathcal{L}}$ from which the eigenfrequencies $\left\{f_{\alpha}^{\mathcal{L}}\right\}_{\alpha}$ associated with the LF-scale modes $\left\{\boldsymbol{\varphi}_{\alpha}^{\mathcal{L}}\right\}_{\alpha}$ are written as $f_{\alpha}^{\mathcal{L}}=\sqrt{\lambda_{\alpha}^{\mathcal{L}}} / 2 \pi$. The $n_{\mathcal{L}}$ LF-scale modes $\left\{\varphi_{\alpha}^{\mathcal{L}}\right\}_{\alpha}$ constitute an orthogonal vector basis (with respect to $[\mathbb{K}]$ ) of $\mathcal{S}_{\mathcal{L}}$ that is a subspace of $\mathcal{S}_{\text {glob. }}$ As $\left[\Psi^{\mathcal{L}}\right]=\left[\Phi^{g}\right]\left[S^{\mathcal{L}}\right]$ and considering Eq. (32), it can be seen that the generalized coordinates $\left\{\mathbf{w}_{\alpha}^{\mathcal{L}}\right\}_{\alpha}$ such that $\boldsymbol{\varphi}_{\alpha}^{\mathcal{L}}=\left[\Phi^{g}\right] \mathbf{w}_{\alpha}^{\mathcal{L}}$ are given by $\mathbf{w}^{\mathcal{L}, \alpha}=\left[S^{\mathcal{L}}\right] \mathbf{r}_{\alpha}^{\mathcal{L}}$. Let $\left[\Phi^{\mathcal{L}}\right]=\left[\boldsymbol{\varphi}_{1}^{\mathcal{L}} \ldots \boldsymbol{\varphi}_{n_{\mathcal{L}}}^{\mathcal{L}}\right]$ 
be the $\left(m \times n_{\mathcal{L}}\right)$ real matrix made up of the LF-scale modal basis. Let $\left[W^{\mathcal{L}}\right]=$ $\left[\mathbf{w}_{1}^{\mathcal{L}} \ldots \mathbf{w}_{n_{\mathcal{L}}}^{\mathcal{L}}\right]$ be the $\left(n_{g} \times n_{\mathcal{L}}\right)$ real matrix such that

$$
\left[\Phi^{\mathcal{L}}\right]=\left[\Phi^{g}\right]\left[W^{\mathcal{L}}\right] .
$$

These matrices will be used for constructing the MF- and HF-scale bases and the matrices of the multilevel ROM.

\subsubsection{MF-scale basis}

The MF-scale modes $\left\{\boldsymbol{\varphi}_{\beta}^{\mathcal{M}}\right\}_{\beta}$ are constructed using approximation subspace $\mathcal{A}_{\mathcal{M}}$ whose associated reduced kinematics allows for obtaining a good representation for the LF and MF scales of displacements. Vector subspace $\mathcal{S}_{\mathcal{M}}=$ $\operatorname{span}\left\{\varphi_{1}^{\mathcal{M}}, \ldots, \varphi_{n_{\mathcal{M}}}^{\mathcal{M}}\right\}$ is constructed in order that it does not intersect $\mathcal{S}_{\mathcal{L}}$. Similarly to the construction of the LF-scale eigenvectors presented in Section 5.1.1, the MF-scale eigenvectors $\left\{\boldsymbol{\psi}_{\beta}^{\mathcal{M}}\right\}_{\beta}$ are represented in $\mathcal{S}_{\text {glob }}$ by

$$
\boldsymbol{\psi}_{\beta}^{\mathcal{M}}=\left[\Phi^{g}\right] \mathbf{s}_{\beta}^{\mathcal{M}},
$$

in which the $n_{g}$-dimensional real vectors $\left\{\mathbf{s}_{\beta}^{\mathcal{M}}\right\}_{\beta}$ are the solutions of the smalldimension generalized eigenvalue problem

$$
\left[\Lambda^{g}\right] \mathbf{s}_{\beta}^{\mathcal{M}}=\sigma_{\beta}^{\mathcal{M}}\left[M_{\mathcal{A}_{\mathcal{M}}}\right] \mathbf{s}_{\beta}^{\mathcal{M}},
$$

in which $\left[M_{\mathcal{A}_{\mathcal{M}}}\right]$ is a positive-semidefinite symmetric $\left(n_{g} \times n_{g}\right)$ real matrix which is written as

$$
\left[M_{\mathcal{A}_{\mathcal{M}}}\right]=\left[\Phi^{g}\right]^{T}\left[\mathbb{M}_{\mathcal{A}_{\mathcal{M}}}\right]\left[\Phi^{g}\right]
$$

The rank $\mathcal{N}_{\mathcal{M}}$ of matrix $\left[M_{\mathcal{A}_{\mathcal{M}}}\right]$ satisfies the inequalities $\mathcal{N}_{\mathcal{M}} \leqslant N_{\mathcal{M}}$ and $\mathcal{N}_{\mathcal{M}} \leqslant$ $n_{g}$ in which $N_{\mathcal{M}}$ is the rank of matrix $\left[\mathbb{M}_{\mathcal{A}_{\mathcal{M}}}\right]$. Let $\left[S^{\mathcal{M}}\right]=\left[\mathbf{s}_{1}^{\mathcal{M}} \ldots \mathbf{s}_{\nu_{\mathcal{M}}^{\mathcal{M}}}^{\mathcal{M}}\right]$ be the $\left(n_{g} \times \nu_{\mathcal{M}}\right)$ real matrix constituted of the eigenvectors $\left\{\mathbf{s}_{\beta}^{\mathcal{M}}\right\}_{\beta}$ associated with the first $\nu_{\mathcal{M}}$ finite positive eigenvalues $0<\sigma_{1}^{\mathcal{M}} \leqslant \ldots \leqslant \sigma_{\nu_{\mathcal{M}}^{\mathcal{M}}}^{\mathcal{M}}$. The matrix $\left\{\boldsymbol{\psi}_{\beta}^{\mathcal{M}}\right\}_{\beta}$ of the MF-scale eigenvectors is written as

$$
\left[\Psi^{\mathcal{M}}\right]=\left[\Phi^{g}\right]\left[S^{\mathcal{M}}\right] .
$$

The $\nu_{\mathcal{M}}$ MF-scale modes $\left\{\boldsymbol{\varphi}_{\beta}^{\mathcal{M}}\right\}_{\beta}$ are then defined by

$$
\varphi_{\beta}^{\mathcal{M}}=\left[\Psi^{\mathcal{M}}\right] \mathbf{v}_{\beta}^{\mathcal{M}},
$$


in which $\left\{\mathbf{v}_{\beta}^{\mathcal{M}}\right\}_{\beta}$ are $\nu_{\mathcal{M}}$-dimensional real vectors. In order that $\mathcal{S}_{\mathcal{M}}$ does not intersect $\mathcal{S}_{\mathcal{L}}$, the MF-scale modes $\left\{\boldsymbol{\varphi}_{\beta}^{\mathcal{M}}\right\}_{\beta}$ are constructed under the constraint of being orthogonal to $\mathcal{S}_{\mathcal{L}}$ (with respect to $[\mathbb{K}]$ ), which means that for all $\beta=$ $1, \ldots, n_{\mathcal{M}}\left(n_{\mathcal{M}}\right.$ will be defined after), we have

$$
\left[\Phi^{\mathcal{L}}\right]^{T}[\mathbb{K}] \boldsymbol{\varphi}_{\beta}^{\mathcal{M}}=\mathbf{0}
$$

From Eqs. (39) and (40), it can be deduced that, for all $\beta=1, \ldots, n_{\mathcal{M}}$,

$$
\left[C_{\mathcal{M}}\right] \mathbf{v}_{\beta}^{\mathcal{M}}=\mathbf{0}
$$

in which the $\left(n_{\mathcal{L}} \times \nu_{\mathcal{M}}\right)$ real matrix $\left[C_{\mathcal{M}}\right]=\left[\Phi^{\mathcal{L}}\right]^{T}[\mathbb{K}]\left[\Psi^{\mathcal{M}}\right]$ can be rewritten, using Eqs. (34) and (38), as

$$
\left[C_{\mathcal{M}}\right]=\left[W^{\mathcal{L}}\right]^{T}\left[\Lambda^{g}\right]\left[S^{\mathcal{M}}\right] .
$$

The $n_{\mathcal{M}}$ vectors $\left\{\mathbf{v}_{\beta}^{\mathcal{M}}\right\}_{\beta}$ satisfying Eq. (41) belong to the null space of $\left[C_{\mathcal{M}}\right]$. Let $\left[Z_{\mathcal{M}}\right]$ be a $\left(\nu_{\mathcal{M}} \times n_{\mathcal{M}}\right)$ real matrix made up of a vector basis of the null space of $\left[C_{\mathcal{M}}\right]$. Dimension $n_{\mathcal{M}}$ is thus given by

$$
n_{\mathcal{M}}=\nu_{\mathcal{M}}-n_{\mathcal{L}} \quad, \quad n_{\mathcal{M}} \leqslant n_{g}
$$

and the $n_{\mathcal{M}}$ vectors $\left\{\mathbf{v}_{\beta}^{\mathcal{M}}\right\}_{\beta}$ are written as

$$
\mathbf{v}_{\beta}^{\mathcal{M}}=\left[Z_{\mathcal{M}}\right] \mathbf{r}_{\beta}^{\mathcal{M}}
$$

with $\mathbf{r}_{\beta}^{\mathcal{M}}$ an $n_{\mathcal{M}}$-dimensional real vector. Substituting Eq. (44) into the right-hand side of Eq. (39) and using Eq. (38) yield $\varphi_{\beta}^{\mathcal{M}}=\left[\Phi^{g}\right]\left[S^{\mathcal{M}}\right]\left[Z_{\mathcal{M}}\right] \mathbf{r}_{\beta}^{\mathcal{M}}$, which can be rewritten as

$$
\boldsymbol{\varphi}_{\beta}^{\mathcal{M}}=\left[\Phi^{g}\right]\left[S_{Z}^{\mathcal{M}}\right] \mathbf{r}_{\beta}^{\mathcal{M}}
$$

in which $\left[S_{Z}^{\mathcal{M}}\right]=\left[S^{\mathcal{M}}\right]\left[Z_{\mathcal{M}}\right]$ is a $\left(n_{g} \times n_{\mathcal{M}}\right)$ real matrix. Substituting this last representation of $\varphi_{\beta}^{\mathcal{M}}$ in the usual generalized eigenvalue problem defined by Eq. (2) yields the following small-dimension generalized eigenvalue problem

$$
\left[K_{Z}^{\mathcal{M}}\right] \mathbf{r}_{\beta}^{\mathcal{M}}=\lambda_{\beta}^{\mathcal{M}}\left[M_{Z}^{\mathcal{M}}\right] \mathbf{r}_{\beta}^{\mathcal{M}}
$$


in which $\left[K_{Z}^{\mathcal{M}}\right]$ and $\left[M_{Z}^{\mathcal{M}}\right]$ are positive-definite symmetric $\left(n_{\mathcal{M}} \times n_{\mathcal{M}}\right)$ real matrices such that $\left[K_{Z}^{\mathcal{M}}\right]=\left[S_{\mathbf{Z}}^{\mathcal{M}}\right]^{T}\left[\Lambda^{g}\right]\left[S_{\mathrm{Z}}^{\mathcal{M}}\right]$ and $\left[M_{Z}^{\mathcal{M}}\right]=\left[S_{\mathrm{Z}}^{\mathcal{M}}\right]^{T}\left[S_{\mathrm{Z}}^{\mathcal{M}}\right]$. This generalized eigenvalue problem admits $n_{\mathcal{M}}$ positive eigenvalues $0<\lambda_{1}^{\mathcal{M}} \leqslant \ldots \leqslant \lambda_{n_{\mathcal{M}}}^{\mathcal{M}}$ from which the eigenfrequencies $\left\{f_{\beta}^{\mathcal{M}}\right\}_{\beta}$ associated with the MF-scale modes $\left\{\boldsymbol{\varphi}_{\beta}^{\mathcal{M}}\right\}_{\beta}$ are given by $f_{\beta}^{\mathcal{M}}=\sqrt{\lambda_{\beta}^{\mathcal{M}}} / 2 \pi$. The $n_{\mathcal{M}}$ MF-scale modes $\left\{\boldsymbol{\varphi}_{\beta}^{\mathcal{M}}\right\}_{\beta}$ form an orthogonal vector basis (with respect to $[\mathbb{K}]$ ) of $\mathcal{S}_{\mathcal{M}}$, which is a subspace of

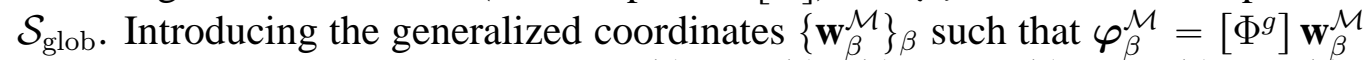
and comparing with Eq. (45) yields $\mathbf{w}_{\beta}^{\mathcal{M}}=\left[S_{\mathrm{Z}}^{\mathcal{M}}\right] \mathbf{r}_{\beta}^{\mathcal{M}}$. Let $\left[\Phi^{\mathcal{M}}\right]=\left[\boldsymbol{\varphi}_{1}^{\mathcal{M}} \ldots \varphi_{n_{\mathcal{M}}}^{\mathcal{M}}\right]$ be the $\left(m \times n_{\mathcal{M}}\right)$ real matrix constituted of the MF-scale modal basis. Let $\left[W^{\mathcal{M}}\right]=$ $\left[\mathbf{w}_{1}^{\mathcal{M}} \ldots \mathbf{w}_{n_{\mathcal{M}}}^{\mathcal{M}}\right]$ be the $\left(n_{g} \times n_{\mathcal{M}}\right)$ real matrix such that

$$
\left[\Phi^{\mathcal{M}}\right]=\left[\Phi^{g}\right]\left[W^{\mathcal{M}}\right] .
$$

These matrices will be used for constructing the HF-scale basis and the matrices of the multilevel ROM.

\subsubsection{HF-scale basis}

The $n_{\mathcal{H}}$ HF-scale modes $\left\{\boldsymbol{\varphi}_{\gamma}^{\mathcal{H}}\right\}_{\gamma}$ allow for obtaining the representation for the remaining contribution of the displacements, which belongs to $\mathcal{S}_{\text {glob }} \backslash\left(\mathcal{S}_{\mathcal{L}} \cup \mathcal{S}_{\mathcal{M}}\right)$. As $\varphi_{\gamma}^{\mathcal{H}}$ belongs to $\mathcal{S}_{\text {glob }}$, it can be written as

$$
\boldsymbol{\varphi}_{\gamma}^{\mathcal{H}}=\left[\Phi^{g}\right] \mathbf{w}_{\gamma}^{\mathcal{H}}
$$

in which $\mathbf{w}_{\gamma}^{\mathcal{H}}$ is an $n_{g}$-dimensional real vector. The dimension $n_{\mathcal{H}}$ of vector subspace $\mathcal{S}_{\mathcal{H}}=\operatorname{span}\left\{\varphi_{1}^{\mathcal{H}}, \ldots, \varphi_{n_{\mathcal{H}}}^{\mathcal{H}}\right\}$ is such that

$$
n_{\mathcal{H}}=n_{g}-n_{\mathcal{L}}-n_{\mathcal{M}},
$$

and consequently, $n_{g} \geqslant n_{\mathcal{H}}$. Similarly to the construction introduced for $\mathcal{S}_{\mathcal{M}}$, subspace $\mathcal{S}_{\mathcal{H}}$ must not intersect $\mathcal{S}_{\mathcal{L}} \cup \mathcal{S}_{\mathcal{M}}$ and consequently, $\varphi_{\gamma}^{\mathcal{H}}$ must satisfy, for all $\gamma=1, \ldots, n_{\mathcal{H}}$,

$$
\left[\Phi^{\mathcal{L M}}\right]^{T}[\mathbb{K}] \boldsymbol{\varphi}_{\gamma}^{\mathcal{H}}=\mathbf{0}
$$

in which the $\left(m \times n_{\mathcal{L M}}\right)$ real matrix $\left[\Phi^{\mathcal{L M}}\right]$ is given (using block writing) as

$$
\left[\Phi^{\mathcal{L} \mathcal{M}}\right]=\left[\left[\Phi^{\mathcal{L}}\right]\left[\Phi^{\mathcal{M}}\right]\right]
$$


with $n_{\mathcal{L M}}=n_{\mathcal{L}}+n_{\mathcal{M}}$. From Eqs. (34) and (47), and introducing the $\left(n_{g} \times\right.$ $n_{\mathcal{L M}}$ ) real matrix $\left[W^{\mathcal{L} \mathcal{M}}\right]=\left[\left[W^{\mathcal{L}}\right]\left[W^{\mathcal{M}}\right]\right]$ (block writing), matrix $\left[\Phi^{\mathcal{L M}}\right]$ that is defined by Eq. (51) can be rewritten as

$$
\left[\Phi^{\mathcal{L M}}\right]=\left[\Phi^{g}\right]\left[W^{\mathcal{L M}}\right]
$$

Substituting Eq. (48) into Eq. (50) yields $\left[\Phi^{\mathcal{L} \mathcal{M}}\right]^{T}[\mathbb{K}]\left[\Phi^{g}\right] \mathbf{w}_{\gamma}^{\mathcal{H}}=\mathbf{0}$. Substituting Eq. (52) into this last equation and using $\left[\Lambda^{g}\right]=\left[\Phi^{g}\right]^{T}[\mathbb{K}]\left[\Phi^{g}\right]$ yield, for all $\gamma=1, \ldots, n_{\mathcal{H}}$,

$$
\left[C_{\mathcal{H}}\right] \mathbf{w}_{\gamma}^{\mathcal{H}}=\mathbf{0}
$$

in which the $\left(n_{\mathcal{L M}} \times n_{g}\right)$ real matrix $\left[C_{\mathcal{H}}\right]$ is written as

$$
\left[C_{\mathcal{H}}\right]=\left[W^{\mathcal{L M}}\right]^{T}\left[\Lambda^{g}\right]
$$

From Eq. (53), it can be deduced that the $n_{\mathcal{H}}$ vectors $\left\{\mathbf{w}_{\gamma}^{\mathcal{H}}\right\}_{\gamma}$ must belong to the null space of $\left[C_{\mathcal{H}}\right]$. Let $\left[Z_{\mathcal{H}}\right]$ be a $\left(n_{g} \times n_{\mathcal{H}}\right)$ real matrix made up of a vector basis of the null space of $\left[C_{\mathcal{H}}\right]$. The $n_{\mathcal{H}}$ vectors $\left\{\mathbf{w}_{\gamma}^{\mathcal{H}}\right\}_{\gamma}$ are thus written as

$$
\mathbf{w}_{\gamma}^{\mathcal{H}}=\left[Z_{\mathcal{H}}\right] \mathbf{r}_{\gamma}^{\mathcal{H}}
$$

in which $\left\{\mathbf{r}_{\gamma}^{\mathcal{H}}\right\}_{\gamma}$ are $n_{\mathcal{H}}$-dimensional real vectors. From Eqs. (48) and (55), it can be deduced that

$$
\boldsymbol{\varphi}_{\gamma}^{\mathcal{H}}=\left[\Phi^{g}\right]\left[Z_{\mathcal{H}}\right] \mathbf{r}_{\gamma}^{\mathcal{H}}
$$

Substituting Eq. (56) into Eq. (2) and left multiplying the resulting equation by $\left[Z_{\mathcal{H}}\right]^{T}\left[\Phi^{g}\right]^{T}$ yield the small-dimension generalized eigenvalue problem

$$
\left[K_{Z}^{\mathcal{H}}\right] \mathbf{r}_{\gamma}^{\mathcal{H}}=\lambda_{\gamma}^{\mathcal{H}}\left[M_{Z}^{\mathcal{H}}\right] \mathbf{r}_{\gamma}^{\mathcal{H}},
$$

in which $\left[K_{Z}^{\mathcal{H}}\right]$ and $\left[M_{Z}^{\mathcal{H}}\right]$ are positive-definite symmetric $\left(n_{\mathcal{H}} \times n_{\mathcal{H}}\right)$ real matrices that are such that $\left[K_{Z}^{\mathcal{H}}\right]=\left[Z_{\mathcal{H}}\right]^{T}\left[\Lambda^{g}\right]\left[Z_{\mathcal{H}}\right]$ and $\left[M_{Z}^{\mathcal{H}}\right]=\left[Z_{\mathcal{H}}\right]^{T}\left[Z_{\mathcal{H}}\right]$. This generalized eigenvalue problem admits $n_{\mathcal{H}}$ real eigenvalues $0<\lambda_{1}^{\mathcal{H}} \leqslant \ldots \leqslant \lambda_{n_{\mathcal{H}}}^{\mathcal{H}}$ from which the eigenfrequencies $\left\{f_{\gamma}^{\mathcal{H}}\right\}_{\gamma}$ associated with the HF-scale modes $\left\{\varphi_{\gamma}^{\mathcal{H}}\right\}_{\gamma}$ are written as $f_{\gamma}^{\mathcal{H}}=\sqrt{\lambda_{\gamma}^{\mathcal{H}}} / 2 \pi$. The $n_{\mathcal{H}} \mathrm{HF}$-scale modes $\left\{\boldsymbol{\varphi}_{\gamma}^{\mathcal{H}}\right\}_{\gamma}$ constitute an orthogonal vector basis (with respect to $[\mathbb{K}]$ ) of $\mathcal{S}_{\mathcal{H}}$, which is a subspace of $\mathcal{S}_{\text {glob }}$. Let 
$\left[\Phi^{\mathcal{H}}\right]=\left[\boldsymbol{\varphi}_{1}^{\mathcal{H}} \ldots \boldsymbol{\varphi}_{n_{\mathcal{H}}}^{\mathcal{H}}\right]$ be the $\left(m \times n_{\mathcal{H}}\right)$ real matrix made up of the HF-scale modal basis. Let $\left[W^{\mathcal{H}}\right]=\left[\mathbf{w}_{1}^{\mathcal{H}} \ldots \mathbf{w}_{n_{\mathcal{H}}}^{\mathcal{H}}\right]$ be the $\left(n_{g} \times n_{\mathcal{H}}\right)$ real matrix such that

$$
\left[\Phi^{\mathcal{H}}\right]=\left[\Phi^{g}\right]\left[W^{\mathcal{H}}\right]
$$

These matrices will be used for constructing the matrices of the multilevel ROM.

\subsubsection{Construction of the multilevel ROM}

By construction, subspaces $\mathcal{S}_{\mathcal{L}}, \mathcal{S}_{\mathcal{M}}$, and $\mathcal{S}_{\mathcal{H}}$ with dimension $n_{\mathcal{L}}, n_{\mathcal{M}}$, and $n_{\mathcal{H}}$ respectively, are orthogonal subspaces (with respect to $[\mathbb{K}]$ ) of space $\mathcal{S}_{\text {glob }}$ that has dimension $n_{g}$. Since $n_{g}=n_{\mathcal{L}}+n_{\mathcal{M}}+n_{\mathcal{H}}$ (see Eq. (49)), subspaces $\mathcal{S}_{\mathcal{L}}, \mathcal{S}_{\mathcal{M}}$, and $\mathcal{S}_{\mathcal{H}}$ are such that

$$
\mathcal{S}_{\text {glob }}=\mathcal{S}_{\mathcal{L}} \oplus \mathcal{S}_{\mathcal{M}} \oplus \mathcal{S}_{\mathcal{H}}
$$

in which $\oplus$ denotes the direct sum. For all $\omega$ in $\mathcal{B}$, the approximation $\mathbb{U}^{\left(n_{\mathcal{L}}, n_{\mathcal{M}}, n_{\mathcal{H}}\right)}(\omega)$ $\in \mathcal{S}_{\text {glob }}^{c}$ of $\mathbb{U}(\omega)(\mathbb{U}(\omega)$ being the solution of Eq. (1)) is written as

$$
\mathbb{U}^{\left(n_{\mathcal{L}}, n_{\mathcal{M}}, n_{\mathcal{H}}\right)}(\omega)=\left[\Phi^{\mathcal{L}}\right] \mathbf{q}^{\mathcal{L}}(\omega)+\left[\Phi^{\mathcal{M}}\right] \mathbf{q}^{\mathcal{M}}(\omega)+\left[\Phi^{\mathcal{H}}\right] \mathbf{q}^{\mathcal{H}}(\omega)
$$

where the complex vectors $\mathbf{q}^{\mathcal{L}}(\omega), \mathbf{q}^{\mathcal{M}}(\omega)$, and $\mathbf{q}^{\mathcal{H}}(\omega)$ with dimension $n_{\mathcal{L}}, n_{\mathcal{M}}$, and $n_{\mathcal{H}}$ respectively, are associated with the LF-, MF-, and HF-scales. Equation (60) can be rewritten as

$$
\mathbb{U}^{\left(n_{\mathcal{L}}, n_{\mathcal{M}}, n_{\mathcal{H}}\right)}(\omega)=\left[\Phi^{\mathcal{L M H}}\right] \mathbf{q}^{\mathcal{L M \mathcal { H }}}(\omega)
$$

in which $\mathbf{q}^{\mathcal{L} \mathcal{H}}(\omega)=\left(\mathbf{q}^{\mathcal{L}}(\omega), \mathbf{q}^{\mathcal{M}}(\omega), \mathbf{q}^{\mathcal{H}}(\omega)\right)$ is an $n_{g}$-dimensional complex vector and where $\left[\Phi^{\mathcal{L M H}}\right]=\left[\left[\Phi^{\mathcal{L}}\right]\left[\Phi^{\mathcal{M}}\right]\left[\Phi^{\mathcal{H}}\right]\right]$ is a $\left(m \times n_{g}\right)$ real matrix (defined by a block writing). From Eqs. (2) and (61), it can be deduced that

$$
\left(-\omega^{2}[M]+i \omega[D]+[K]\right) \mathbf{q}^{\mathcal{L M H}}(\omega)=\mathcal{F}^{\mathcal{L M H}}(\omega),
$$

in which $\mathcal{F}^{\mathcal{L M H}}(\omega)=\left[\Phi^{\mathcal{L} \mathcal{M H}}\right]^{T} \mathbb{F}(\omega)$ and where, for $(A, \mathbb{A}) \in\{(M, \mathbb{M}),(D, \mathbb{D})$, $(K, \mathbb{K})\}$, we have $[A]=\left[\Phi^{\mathcal{L} \mathcal{M H}}\right]^{T}[\mathbb{A}]\left[\Phi^{\mathcal{L M H}}\right]$. It can be seen that matrix $[A]$ can be rewritten (block writing) as

$$
[A]=\left[\begin{array}{lll}
{\left[A^{\mathcal{L L}}\right]} & {\left[A^{\mathcal{L M}}\right]} & {\left[A^{\mathcal{L H}}\right]} \\
{\left[A^{\mathcal{M L}}\right]} & {\left[A^{\mathcal{M M}}\right]} & {\left[A^{\mathcal{M H}}\right]} \\
{\left[A^{\mathcal{H L}}\right]} & {\left[A^{\mathcal{H M}}\right]} & {\left[A^{\mathcal{H H}}\right]}
\end{array}\right],
$$


in which, for $\mathcal{I}$ and $\mathcal{J}$ in $\{\mathcal{L}, \mathcal{M}, \mathcal{H}\},\left[A^{\mathcal{I} \mathcal{J}}\right]=\left[\Phi^{\mathcal{I}}\right]^{T}[\mathbb{A}]\left[\Phi^{\mathcal{J}}\right]$ is a $\left(n_{\mathcal{I}} \times n_{\mathcal{J}}\right)$ real matrix. In the following, the normalization of $\left[\Phi^{\mathcal{I}}\right]$ is chosen such that $\left[M^{\mathcal{I} \mathcal{I}}\right]=$ $\left[I_{n_{\mathcal{I}}}\right]$. Consequently, matrix $[K]$ can be written as

$$
[K]=\left[\begin{array}{ccc}
{\left[\Lambda^{\mathcal{L}}\right]} & {[0]} & {[0]} \\
{[0]} & {\left[\Lambda^{\mathcal{M}}\right]} & {[0]} \\
{[0]} & {[0]} & {\left[\Lambda^{\mathcal{H}}\right]}
\end{array}\right],
$$

in which $\left[\Lambda^{\mathcal{L}}\right],\left[\Lambda^{\mathcal{M}}\right]$, and $\left[\Lambda^{\mathcal{H}}\right]$ are the diagonal matrices of the LF-, MF-, and HFscale eigenvalues $\left\{\lambda_{\alpha}^{\mathcal{L}}\right\}_{\alpha},\left\{\lambda_{\beta}^{\mathcal{M}}\right\}_{\beta}$, and $\left\{\lambda_{\gamma}^{\mathcal{H}}\right\}_{\gamma}$. For optimizing the computational cost, matrices $[M]$ and $[D]$ are rewritten using matrices $\left[W^{\mathcal{L}}\right],\left[W^{\mathcal{M}}\right]$, and $\left[W^{\mathcal{H}}\right]$. From Eqs. (34), (47), and (58), it can be deduced that, for $\mathcal{I}$ and $\mathcal{J}$ in $\{\mathcal{L}, \mathcal{M}, \mathcal{H}\}$, $\left[M^{\mathcal{I} \mathcal{J}}\right]=\left[W^{\mathcal{I}}\right]^{T}\left[W^{\mathcal{J}}\right]$ and $\left[D^{\mathcal{I} \mathcal{J}}\right]=\left[W^{\mathcal{I}}\right]^{T}\left[\mathcal{D}^{g}\right]\left[W^{\mathcal{J}}\right]$, in which $\left[\mathcal{D}^{g}\right]$ has been introduced in Eq. (21).

Remark 1. For all $\omega$ in $\mathcal{B}, \mathbb{U}^{\left(n_{\mathcal{L}}, n_{\mathcal{M}}, n_{\mathcal{H}}\right)}(\omega)$ coincides with $\mathbb{U}^{\left(N_{d}, n_{g}\right)}(\omega)$. The role of the multilevel representation (see Eq. (60)) lies in its ability to adapt the physical models with respect to each type of coordinates $\mathbf{q}^{\mathcal{L}}(\omega), \mathbf{q}^{\mathcal{M}}(\omega)$, and $\mathbf{q}^{\mathcal{H}}(\omega)$. This ROM allows a greater flexibility to be obtained regarding the uncertainty modeling (particularly when using the nonparametric probabilistic approach [27] for which the nominal reduced matrices are replaced by random matrices) as well as regarding the damping modeling.

Remark 2. The multilevel ROM has been constructed using an indirect method, which consists in working in subspace $\mathcal{S}_{\text {glob }}$. If $\left[\Phi^{g}\right]$ is taken as $[\Phi]$ and $\left[\Lambda^{g}\right]$ as $[\Lambda]$ (which means that $\mathcal{S}_{\text {glob }}$ coincides with $\mathcal{S}_{\text {elas }}$ ), then no approximation is introduced in the multilevel ROM, in comparison to a classical modal analysis.

In the next section, we apply the proposed method to an automobile, which is a complex structure, in order to construct an adapted global-displacements ROM, which is then used for constructing a multilevel ROM.

\section{Application to the computational model of an automobile}

This complex structure is made up of a stiff master part on which flexible parts are attached, as it can be seen in Fig. 2 in which the gray intensity is related to the level of rigidity. It can be seen that there are numerous flexible parts spread over the whole structure (not only well identified components such as the roof or the floor panels, but also erratically distributed flexible parts, see for instance the 
parts located at the front of the car). The presence of the flexible parts induces a high modal density. The computational model has $N_{f}=243,783$ free nodes and $m=1,462,698$ DOFs. It contains various types of finite elements such as volume, surface, beam, bar elements, and also rigid body elements. We are interested in predicting the FRFs of the stiff master part in the frequency band $\mathcal{B}=2 \pi \times] 0,500] \mathrm{rad} / \mathrm{s}$. There are $n=1,457$ elastic modes $\left\{\boldsymbol{\varphi}_{\alpha}\right\}_{\alpha}$ below $600 \mathrm{~Hz}$ and 1,048 elastic modes in frequency band $\mathcal{B}$. For $\omega$ in $\mathcal{B}$, response $\mathbb{U}^{(n)}(\omega)$ is computed by using the classical ROM given by Eqs. (3) and (4). The large value of dimension $n$ for the classical ROM is induced by the presence of numerous local elastic modes intertwined with the usual global elastic modes, due to the numerous flexible parts aforementioned. The first elastic mode $\varphi_{1}$ with $f_{1}=24 \mathrm{~Hz}$ is a local elastic mode while the third one $\varphi_{3}$ with $f_{3}=39 \mathrm{~Hz}$ is a global elastic mode (see Fig. 3). In general, each elastic mode cannot be identified either as a pure local elastic mode or as a pure global elastic mode. An example of elastic mode with mixed global and local displacements is shown in Fig 4. Such a mixed mode $\varphi_{18}$ is constituted of a global deformation of the structure assorted with local deformations of distinct structural levels (the roof, the flexible part in the left back). Furthermore, as the frequency increases, the contributions of the global displacements in the elastic modes are becoming less and less perceptible. Most of the mode shapes are dominated by large-amplitude local displacements that are irregularly distributed over the structure, as it can be seen for mode $\varphi_{300}$ in Fig. 4.

It should be noted that Fig. 2 shows two complementary points of view of the automobile in order to put into evidence the stiff part skeleton, Fig. 3 illustrates the key concept of local modes, as opposed to global modes, and finally, Fig. 4 illustrates the facts that the elastic modes are not either purely local or purely global, and that the local displacements become predominant in higher frequencies.
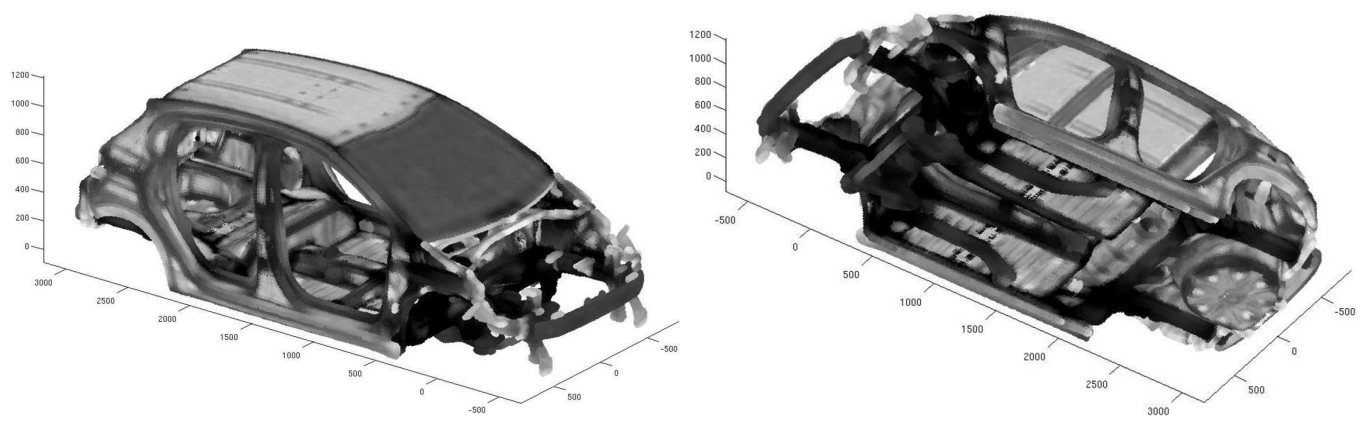

Figure 2: Computational model, in which the gray intensity is related to the level of rigidity (the darker is the stiffer) 

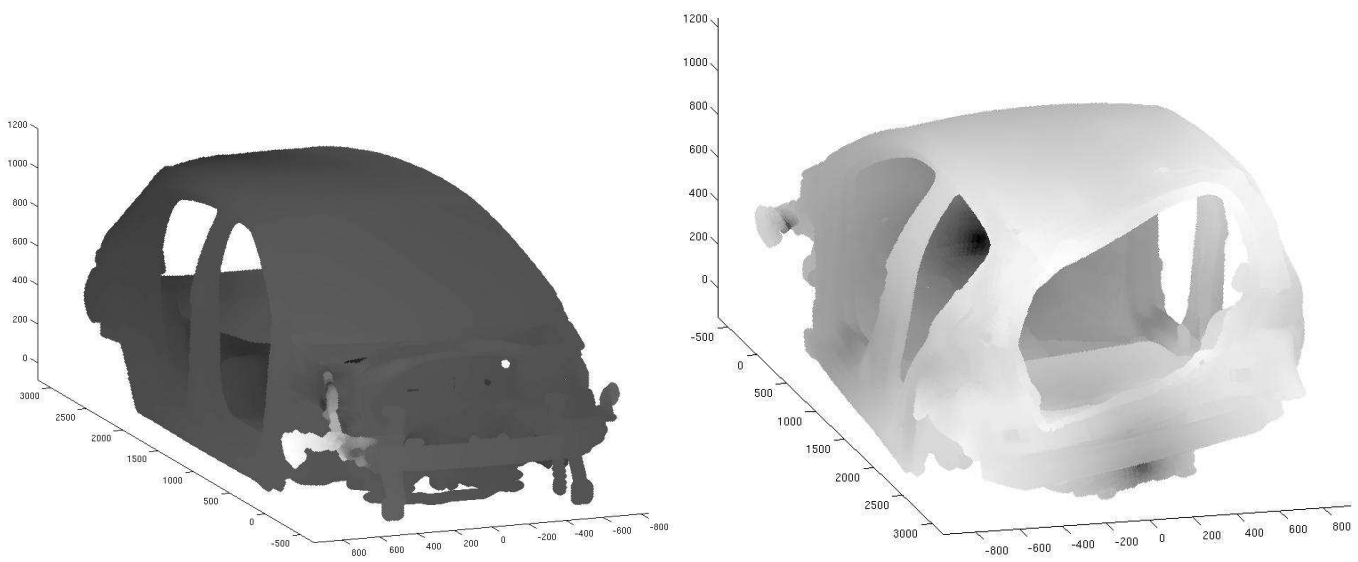

Figure 3: Elastic modes $\varphi_{1}, f_{1}=24 \mathrm{~Hz}$ (left) and $\varphi_{3}, f_{3}=39 \mathrm{~Hz}$ (right), in which the gray intensity is related to the level of amplitude of the displacements (the greater amplitude is the lighter)
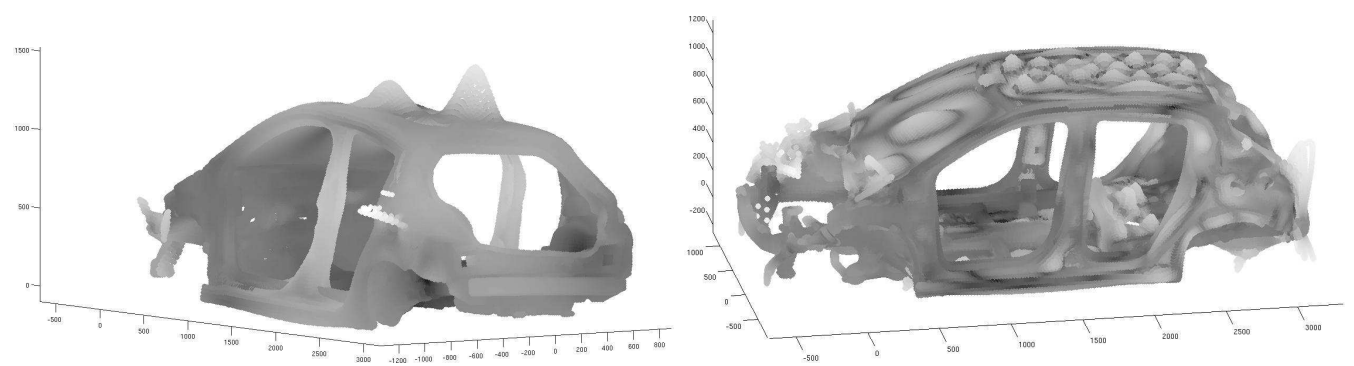

Figure 4: Elastic modes $\varphi_{18}, f_{18}=72 \mathrm{~Hz}$ (left) and $\varphi_{300}, f_{300}=262 \mathrm{~Hz}$ (right), in which the gray intensity is related to the level of amplitude of the displacements (the greater amplitude is the lighter)

\subsection{Implementation of the global-displacements ROM}

The indirect method proposed in Section 4.2 is used for constructing a globaldisplacements ROM of small dimension, given by Eqs. (20) and (21). Subspace $\mathcal{A}_{g}$ that is spanned by multivariate polynomials as presented in Section 3.3, is constructed for an approximation degree $N_{d}$ ranging from 0 to 40 . For instance, the calculated rank of matrix $\left[M_{\mathcal{A}_{g}}\right]$ is $\mathcal{N}_{g}=168$ for $N_{d}=5, \mathcal{N}_{g}=855$ for $N_{d}=$ 10 , and $\mathcal{N}_{g}=n=1,457$ for both $N_{d}=15$ and $N_{d}=20$. In general, for $\mathcal{N}_{g}=n$, it would be possible to compute $n$ global eigenvectors $\left\{\boldsymbol{\psi}_{\alpha}\right\}_{\alpha}$ that would belong (by construction) to subspace $\mathcal{S}_{\text {elas }}$ of dimension $n$, and which would constitute a vector basis of $\mathcal{S}_{\text {elas. }}$. In this case, the $n$ global modes $\left\{\varphi_{\alpha}^{g}\right\}_{\alpha}$ constructed via 
the $n$ solutions $\left\{\mathbf{r}_{\alpha}\right\}_{\alpha}$ of Eq. (19) would coincide with the elastic modes $\left\{\boldsymbol{\varphi}_{\alpha}\right\}_{\alpha}$ and consequently, no gain would be obtained with respect to the dimension of the ROM. The modal density of the global modes thus strongly depends on the truncation order $n_{g}$. In this application, the truncation rule adopted is to set $n_{g}$ as the smallest value such that $f_{n_{g}}^{g} \geqslant 525 \mathrm{~Hz}$. For $N_{d}=5,10,15,20$, the modal density (averaged number of modes per Hz) is plotted in Fig. 5 and is compared to the modal density of the elastic modes. It can be seen that the modal density increases accordingly to the polynomial degree chosen for the reduced kinematics associated with $\mathcal{A}_{g}$.

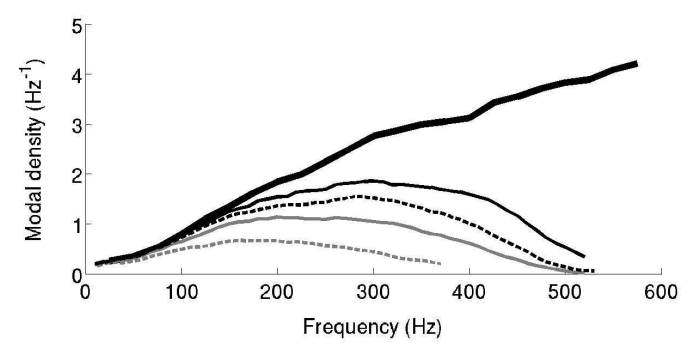

Figure 5: Modal densities: elastic modes (thick black solid line); global modes with: $N_{d}=20$ (thin black solid line), $N_{d}=15$ (thin black dashed line), $N_{d}=10$ (thin gray solid line), and $N_{d}=5$ (thin gray dashed line).

For $N_{d}=5$, the constructed global modes do not cover the whole frequency band of analysis. The dimensions $n_{g}$ of the global-displacements ROM are $n_{g}=$ $168,355,479,624$ for $N_{d}=5,10,15,20$ respectively.

A convergence analysis with respect to $N_{d}$ and $n_{g}$ has been carried out in studying the FRFs. The inputs are unit forces applied in the $x$ - and $z$-directions and unit moments applied around the $x$ - and $y$ - axes, relative to the excitation nodes exc1 and exc2 (see Fig. 6). For $N_{d}$ ranging from 0 to 40, and for all $\omega$ in $\mathcal{B}$, response $\mathbb{U}^{\left(N_{d}, n_{g}\right)}(\omega)$ is computed using the global-displacements ROM given by Eqs. (20) and (21). The outputs are the norm of the displacements vector of 26 observations nodes denoted by $\Gamma_{\mathrm{obs}, \mathrm{dB}}^{\mathrm{ref}}(\omega)=20 \log _{10}\left\|\mathbb{U}_{\mathrm{obs}}^{(n)}\right\|$ for the converged reference and by $\Gamma_{\mathrm{obs}, \mathrm{dB}}^{\mathrm{ROM}}\left(\omega ; n_{g}\right)=20 \log _{10}\left\|\mathbb{U}_{\mathrm{obs}}^{\left(N_{d}, n_{g}\right)}\right\|$ for the computational ROM, among which the FRFs are shown in Fig. 8 to Fig. 11 for the four observation nodes obs1, obs2, obs3, and obs4 whose locations are depicted in Fig. 6. 


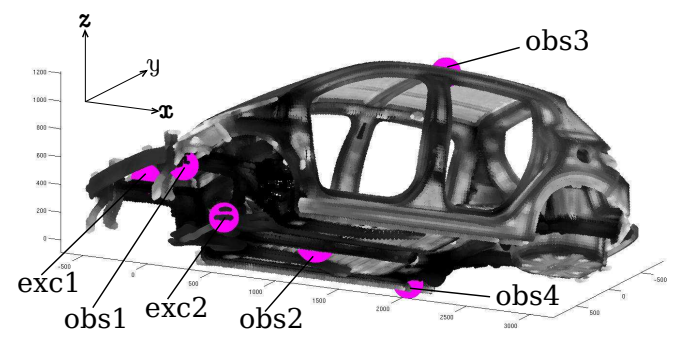

Figure 6: Excitation nodes exc1 and exc2, and observation nodes obs1, obs2, obs3, and obs4.

For the convergence analysis, the error function $\varepsilon_{d B}\left(n_{g}\right)=\sum_{\mathrm{obs}} \varepsilon_{\mathrm{obs}, \mathrm{dB}}\left(n_{g}\right) / n_{\mathrm{obs}}$ is introduced in which the error $\varepsilon_{\mathrm{obs}, \mathrm{dB}}\left(n_{g}\right)$ is such that

$$
\varepsilon_{\mathrm{obs}, \mathrm{dB}}^{2}\left(n_{g}\right)=\frac{\int_{\mathcal{B}}\left(\Gamma_{\mathrm{obs}, \mathrm{dB}}^{\mathrm{ref}}(\omega)-\Gamma_{\mathrm{obs}, \mathrm{dB}}^{\mathrm{ROM}}\left(\omega ; n_{g}\right)\right)^{2} d \omega}{\int_{\mathcal{B}}\left(\Gamma_{\mathrm{obs}, \mathrm{dB}}^{\mathrm{ref}}(\omega)\right)^{2} d \omega},
$$

which depends on dimension $n_{g}$ obtained via $N_{d}$ (following the adopted truncation rule). In Figs. 7 to 11, it can be seen that the error tends to decrease with the increase of $N_{d}$. However, the decrease of the error is accompanied with an increase of dimension $n_{g}$. Consequently, the degree $N_{d}$ as well as the resulting dimension $n_{g}$ must be adjusted regarding the engineering targets. It can be seen that the differences between the reference and the approximated FRFs are larger in higher frequencies, while it is known that the experimental variabilities are also generally larger in higher frequencies.

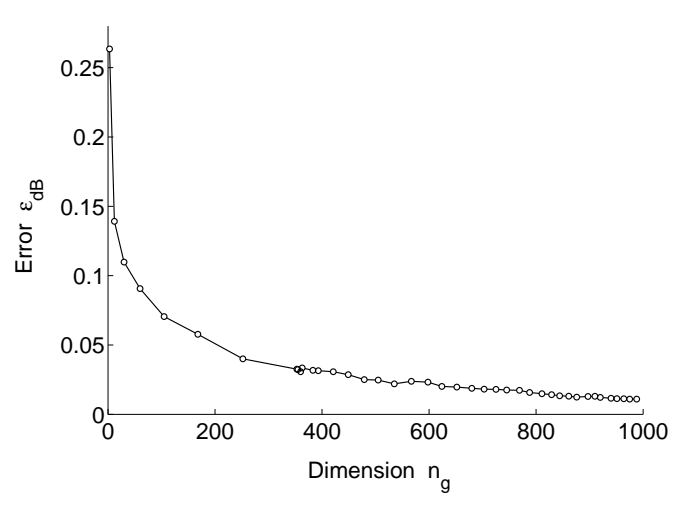

Figure 7: Error $\varepsilon_{\mathrm{dB}}$ with respect to dimension $n_{g}$

For the complex structure studied, it can be seen in Figs. 7 to 11 that a reason- 
able accuracy is obtained for a ROM dimension of about 500 , which has to be compared to the reference-ROM dimension of about 1,500. With respect to the residual errors induced by the use of the proposed global-displacements ROM of reasonable small dimension, it should be noted that the experimental variabilities of the FRFs of such a complex structure [25, 26] are more important than these residual errors.
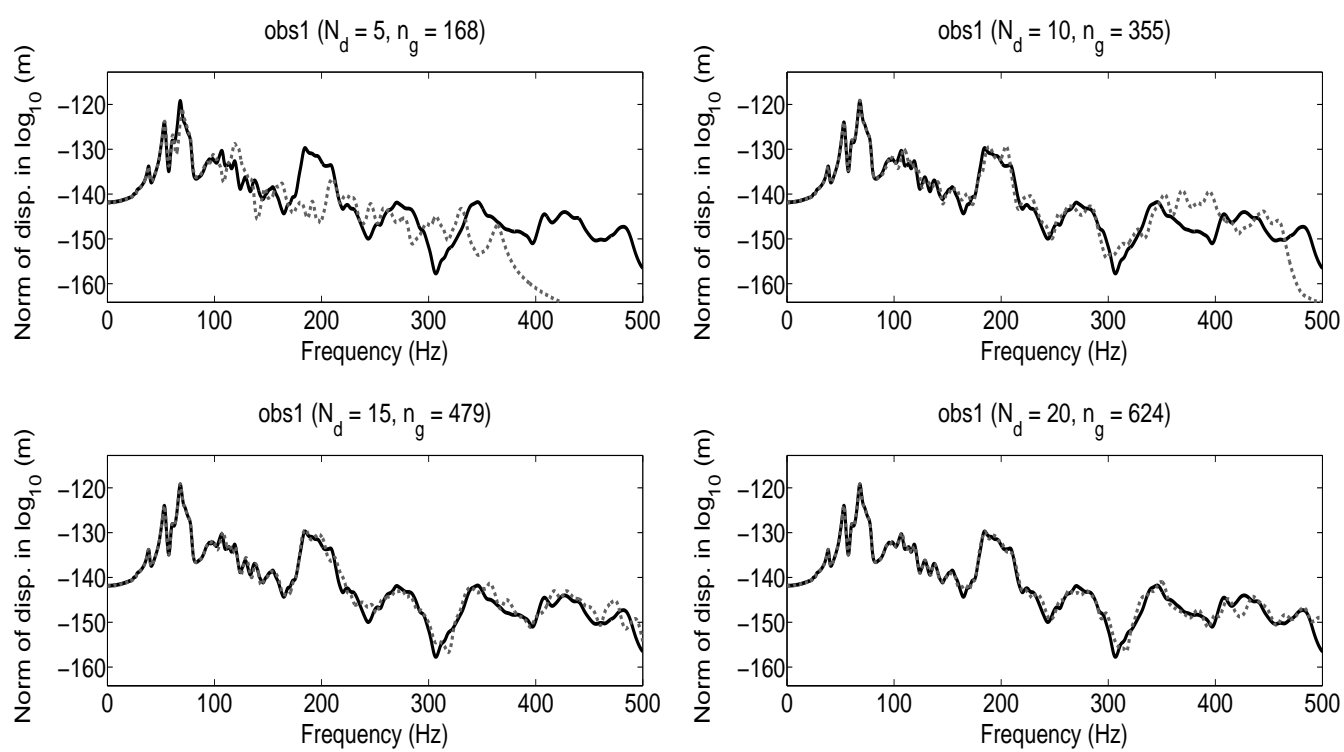

Figure 8: For obs1, logarithm of the norm of the displacements (m) versus frequency (Hz): reference $\mathbb{U}_{\text {obs1 }}^{(n)}$ (black solid line), ROM responses $\left\{\mathbb{U}_{\text {obs1 }}^{\left(N_{d}, n_{g}\right)}\right\}_{N_{d}=5,10,15,20}$ (gray dashed line). 

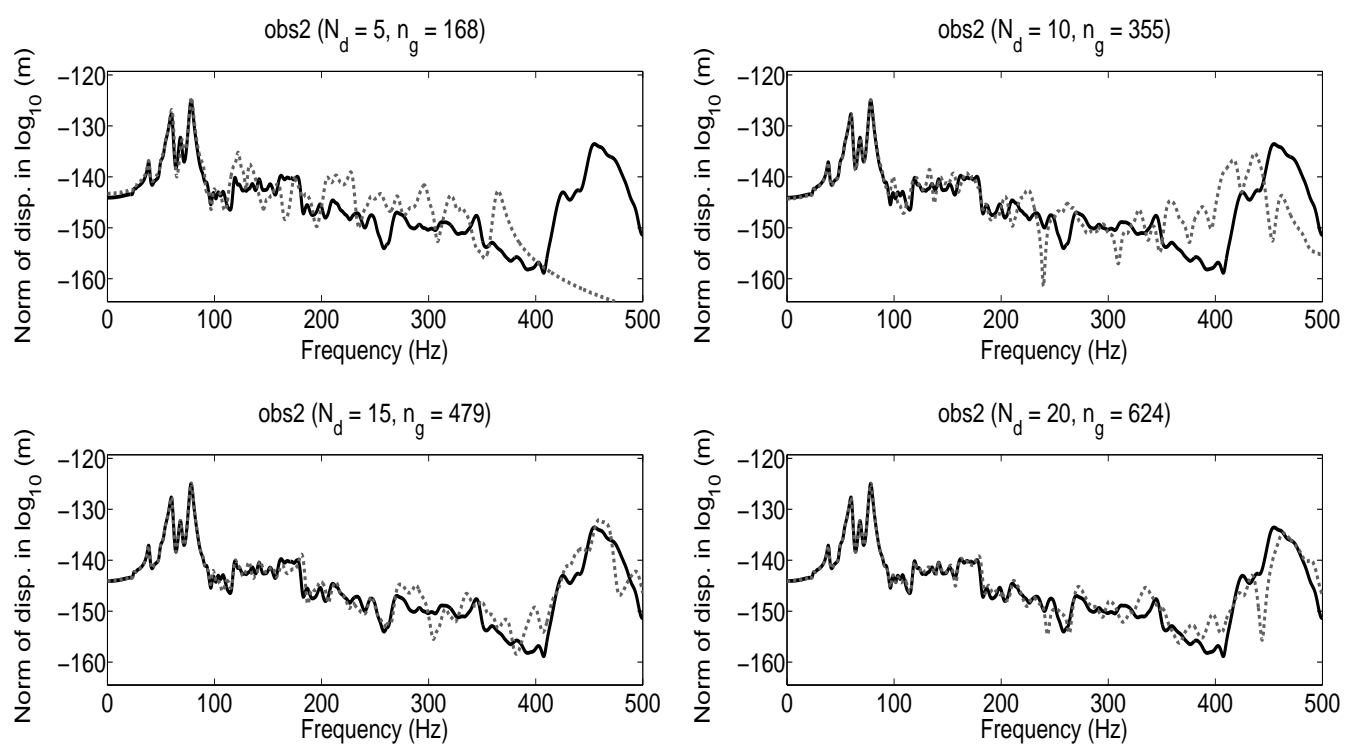

Figure 9: For obs2, logarithm of the norm of the displacements (m) versus frequency (Hz): reference $\mathbb{U}_{\text {obs2 }}^{(n)}$ (black solid line), ROM responses $\left\{\mathbb{U}_{\text {obs2 }}^{\left(N_{d}, n_{g}\right)}\right\}_{N_{d}=5,10,15,20}$ (gray dashed line).
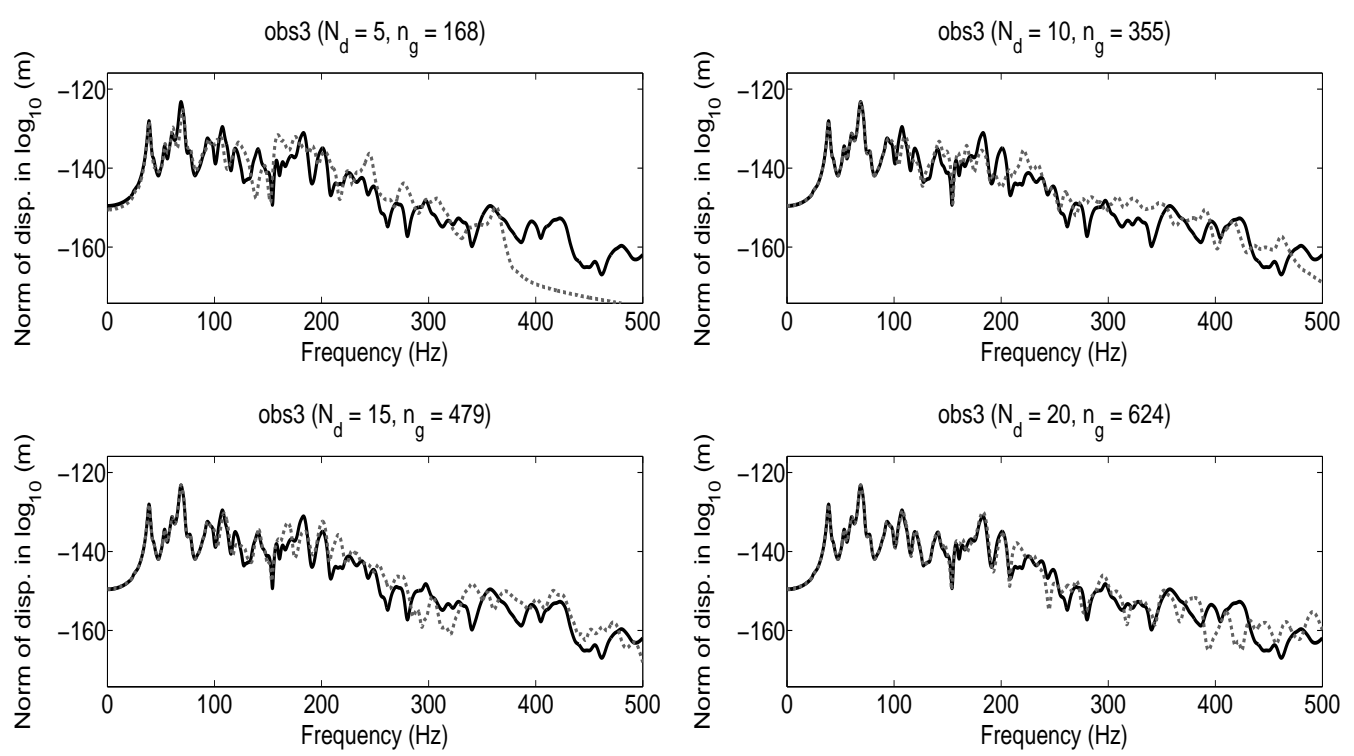

Figure 10: For obs3, logarithm of the norm of the displacements (m) versus frequency $(\mathrm{Hz})$ : reference $\mathbb{U}_{\text {obs3 }}^{(n)}$ (black solid line), ROM responses $\left\{\mathbb{U}_{\text {obs } 3}^{\left(N_{d}, n_{g}\right)}\right\}_{N_{d}=5,10,15,20}$ (gray dashed line). 

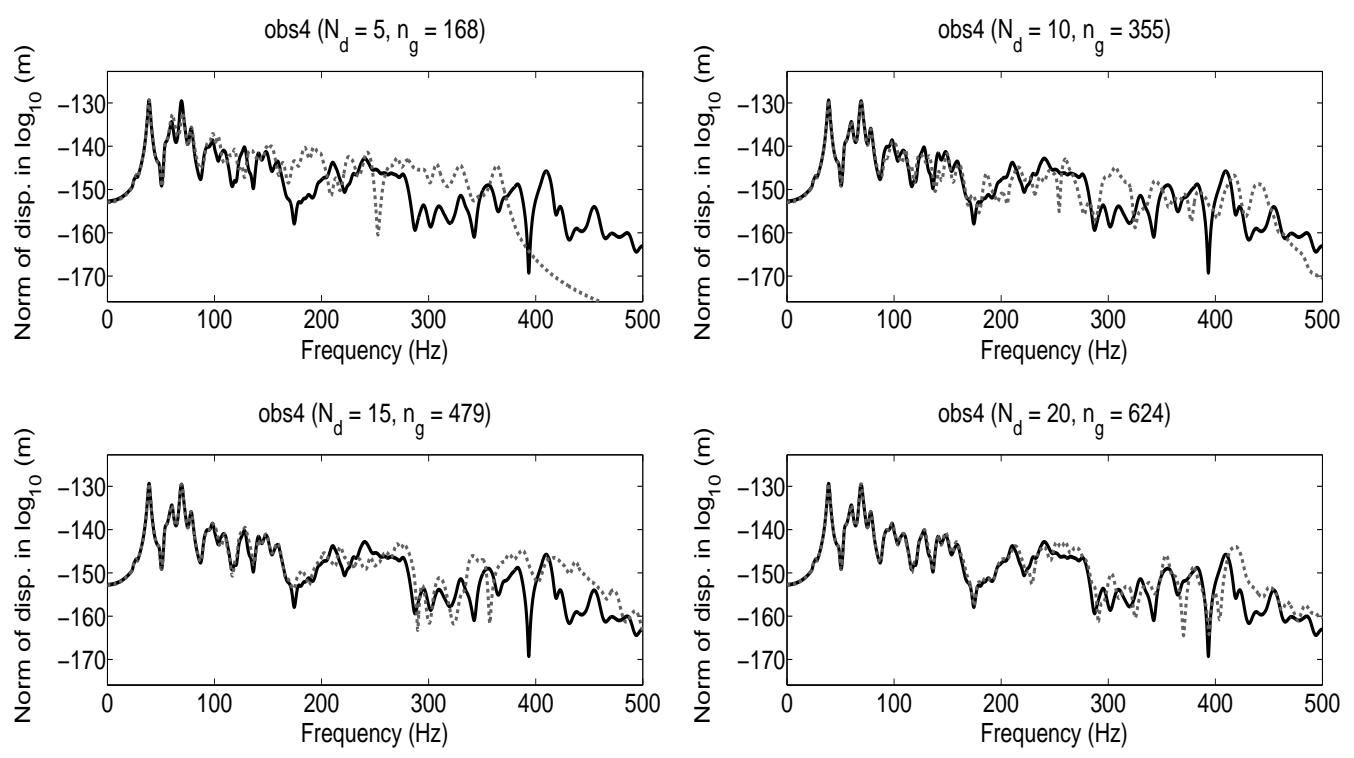

Figure 11: For obs4, logarithm of the norm of the displacements $(\mathrm{m})$ versus frequency $(\mathrm{Hz})$ : reference $\mathbb{U}_{\text {obs } 4}^{(n)}$ (black solid line), ROM responses $\left\{\mathbb{U}_{\text {obs } 4}^{\left(N_{d}, n_{g}\right)}\right\}_{N_{d}=5,10,15,20}$ (gray dashed line).

\subsection{Implementation of a multilevel ROM}

\subsubsection{Multilevel $R O M$ in $\mathcal{S}_{\text {glob }}$}

Based on the previous convergence analysis, $N_{d}=20$ along with $n_{g}=624$ yield a subspace $\mathcal{S}_{\text {glob }}$ associated with a sufficiently accurate global-displacements ROM. In light of the previous results, the methodology presented in Section 5 is applied using subspaces $\mathcal{A}_{\mathcal{L}}$ and $\mathcal{A}_{\mathcal{M}}$ for which $N_{d}=5$ and $N_{d}=15$, respectively. As an example, with $N_{d}=5$, the sharp peaks are well represented in the low frequencies. A more accurate choice of subspaces that define the levels of filtering would be required if a multilevel physical modeling was introduced within the multilevel ROM (by example, for damping and uncertainties). In such a case, a sensitivity analysis would allow for better choosing the degrees.

$L F$-scale basis. The calculated rank of matrix $\left[M_{\mathcal{A}_{\mathcal{L}}}\right]$ is $\mathcal{N}_{\mathcal{L}}=168$. The truncation order $n_{\mathcal{L}}$ is taken as the value of $n_{g}$ obtained in the application of Section 6.1 with $N_{d}=5$. Hence, the $n_{\mathcal{L}}=168$ eigenvectors $\left\{\mathbf{s}_{\alpha}^{\mathcal{L}}\right\}_{\alpha}$ are obtained by solving Eq. (30). The $n_{\mathcal{L}}$ LF-scale modes $\left\{\varphi_{\alpha}^{\mathcal{L}}\right\}_{\alpha}$ are then computed following the method proposed.

$M F$-scale basis. Similarly to the LF-scale basis, the calculated rank of matrix $\left[M_{\mathcal{A}_{\mathcal{M}}}\right]$ is $\mathcal{N}_{\mathcal{M}}=624$ (which corresponds to the value of $n_{g}$ ). The truncation 
order $\nu_{\mathcal{M}}$ is taken as the value of $n_{g}$ obtained in the application of Section 6.1 with $N_{d}=15$. Hence, the first $\nu_{\mathcal{M}}=479$ eigenvectors $\left\{\mathbf{s}_{\beta}^{\mathcal{M}}\right\}_{\beta}$ are computed by using Eq. (36). The $n_{\mathcal{M}}=\nu_{\mathcal{M}}-n_{\mathcal{L}}=311$ MF-scale modes $\left\{\boldsymbol{\varphi}_{\beta}^{\mathcal{M}}\right\}_{\beta}$ are constructed following the method, in which $\left[Z_{\mathcal{M}}\right]$ is made up of the right eigenvectors of the SVD of matrix $\left[C_{\mathcal{M}}\right]$.

$H F$-scale basis. Since $n_{\mathcal{H}}=n_{g}-n_{\mathcal{L}}-n_{\mathcal{M}}$, the HF-scale basis is constituted of $n_{\mathcal{H}}=145$ modes $\left\{\varphi_{\gamma}^{\mathcal{H}}\right\}_{\gamma}$, which are computed following the method.

For each one of these three bases, the modal density is plotted in Fig. 12. It can be seen that none of these bases covers all the frequency band $\mathcal{B}$. Nevertheless, the union of these three bases span $\mathcal{S}_{\text {glob }}$ whose eigenfrequencies cover whole the band $\mathcal{B}$. It should be noted that the modal density of each basis is maximum at the frequency of interest (the maximum is about $180 \mathrm{~Hz}$ for the LF basis, about 280 $\mathrm{Hz}$ for the MF basis, and about $360 \mathrm{~Hz}$ for the HF basis).

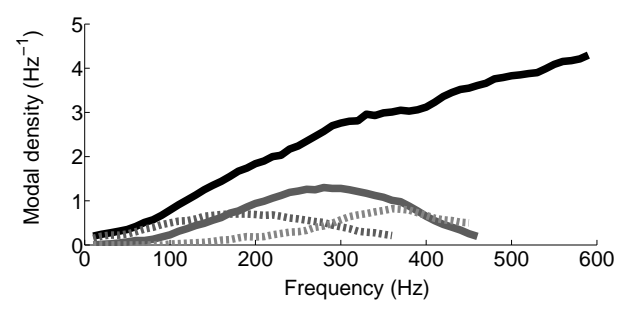

Figure 12: Modal densities: elastic modes ( $n=1,457$; black solid line), LF-scale modes $\left(n_{\mathcal{L}}=\right.$ 168 ; gray dashed line), MF-scale modes ( $n_{\mathcal{M}}=311$; gray solid line), and HF-scale modes $\left(n_{\mathcal{H}}=145 ;\right.$ light-gray dashed line $)$.

Studying the contribution of each scale basis in the responses. For $\mathcal{I}$ in $\{\mathcal{L}, \mathcal{M}, \mathcal{H}\}$, the response $\mathbb{U}^{\mathcal{I}}(\omega)$ is calculated using only the IF-scale modes $\left\{\boldsymbol{\varphi}_{\alpha}^{\mathcal{I}}\right\}_{\alpha}$ represented by matrix $\left[\Phi^{\mathcal{I}}\right]$ and the contributions of each scale basis is plotted in Fig. 13 for observation nodes obs1, obs2, obs3, and obs4. 

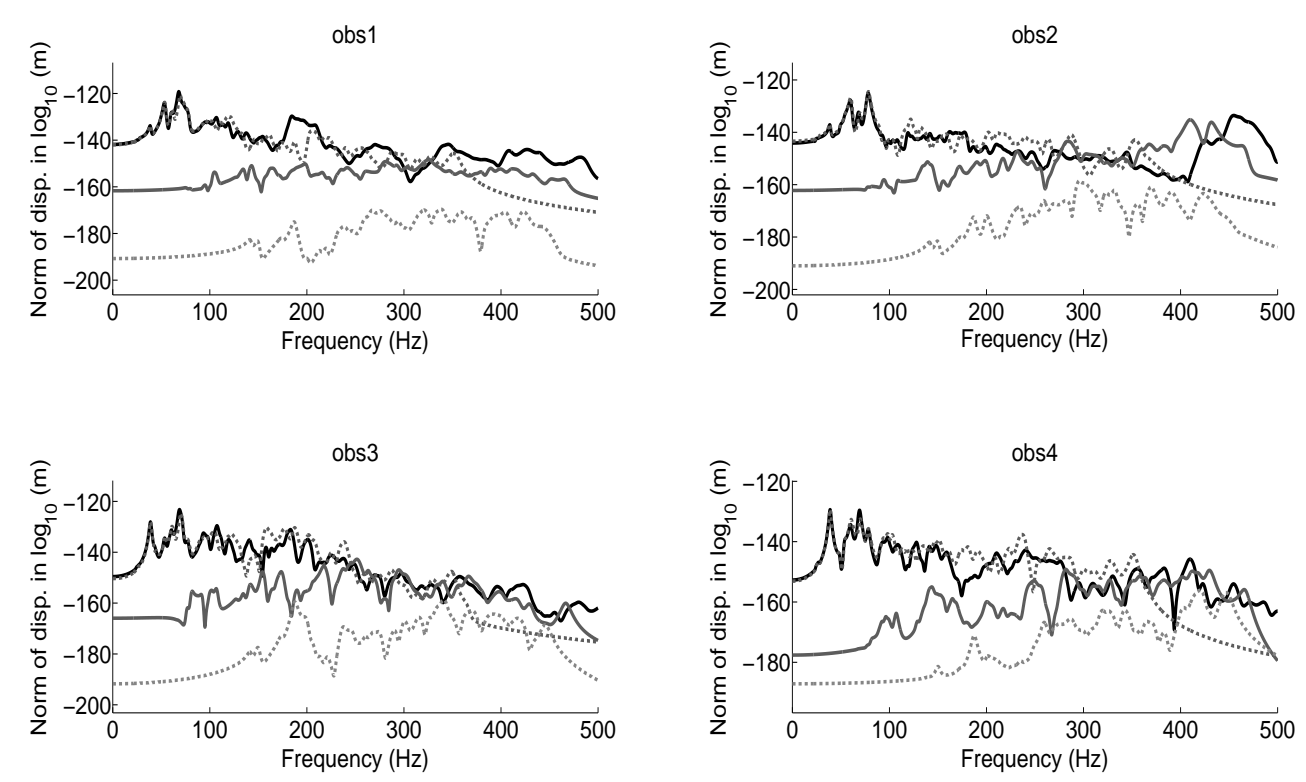

Figure 13: For observation nodes obs1, obs2, obs3, and obs4, graphs of the norm of the displacements in log scale as a function of frequency using: elastic modes (black solid line), LFscale modes (gray dashed line), MF-scale modes (gray solid line), and HF-scale modes (light-gray dashed line).

For each observation node, it can be seen that the amplitude levels of the FRFs that correspond to the three bases are different. The LF-scale basis allows the resonance peaks in low frequencies to be well represented. Furthermore, the level of the FRFs is of the same order than the reference in a large part of the frequency band. In contrast, the MF- and HF-scale bases yield FRFs whose amplitudes are much lower in low frequencies. Nevertheless, the FRFs obtained using the MFscale basis show that the MF-scale modes contribute for an important part in the response as soon as the middle of the frequency band. For the HF-scale modes, the contributions are more important in the high part of the frequency band.

Studying the contribution of combinations of scale bases in the responses. The response $\mathbb{U}^{\mathcal{L M}}(\omega)$ is calculated using both the LF-scale modes $\left\{\boldsymbol{\varphi}_{\alpha}^{\mathcal{L}}\right\}_{\alpha}$ and the MFscale modes $\left\{\boldsymbol{\varphi}_{\beta}^{\mathcal{M}}\right\}_{\beta}$. Figure 14 displays, for observation nodes obs1, obs2, obs3, and obs4, the graphs related to $\mathbb{U}^{\mathcal{L}}(\omega)$ (previously computed), $\mathbb{U}^{\mathcal{L} \mathcal{M}}(\omega)$ (computed as explained hereinbefore), and $\mathbb{U}^{\left(n_{\mathcal{L}}, n_{\mathcal{M}}, n_{\mathcal{H}}\right)}(\omega)$ (computed with Eq. (61)). It can be seen that these FRFs are close to the FRFs related to $\mathbb{U}^{\left(N_{d}, n_{g}\right)}(\omega)$ computed in 
Section 6.1 with Eq. (20) for which $N_{d}=5\left(n_{g}=168\right), N_{d}=15\left(n_{g}=479\right)$, and $N_{d}=20\left(n_{g}=624\right)$.
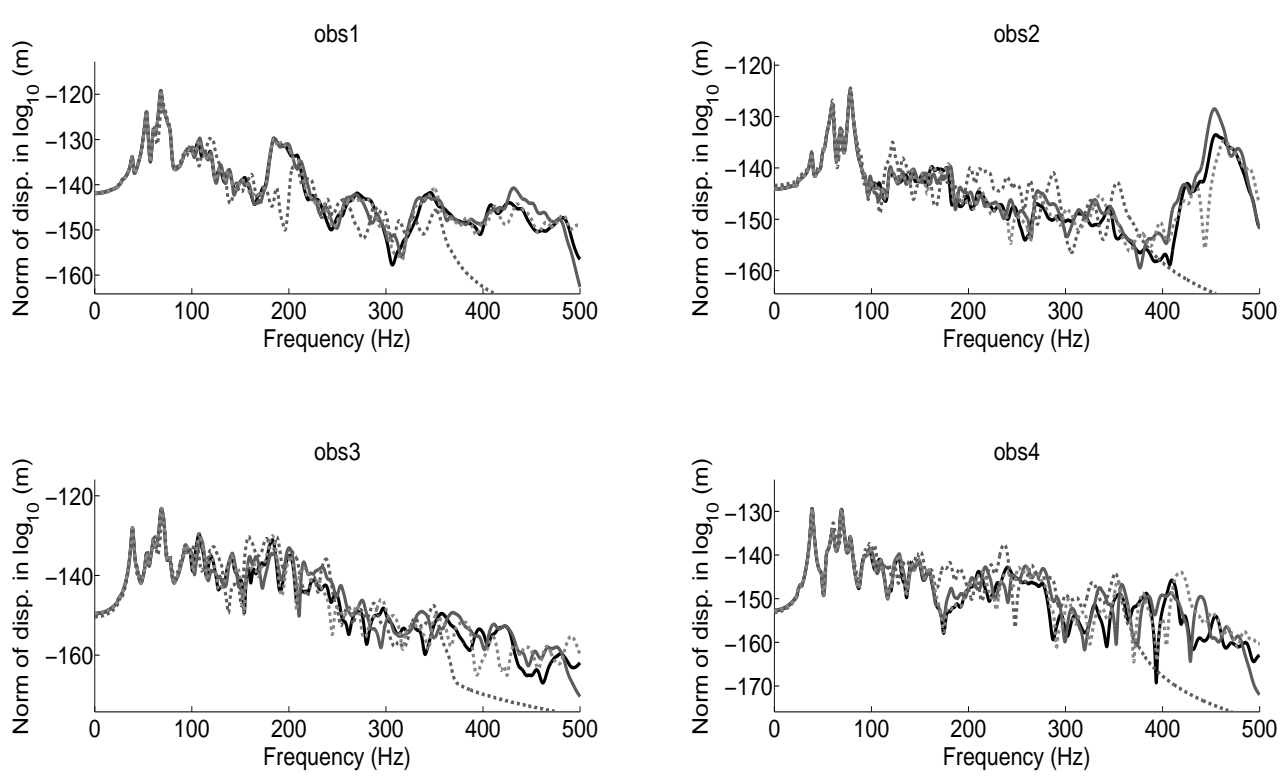

Figure 14: For observation nodes obs1, obs2, obs3, and obs4, graphs of the norm of the displacements in log scale as a function of frequency using: (1) elastic modes (black solid line); (2) LF-scale modes (gray dashed line); (3) LF-scale modes and MF-scale modes (gray solid line); (4) LF-scale modes, MF-scale modes, and HF-scale modes (light-gray dashed line).

\subsubsection{Multilevel ROM in $\mathcal{S}_{\text {elas }}$}

In this last part of the application, the construction of the multilevel ROM is performed in taking $[\Phi]$ for $\left[\Phi^{g}\right]$ and $[\Lambda]$ for $\left[\Lambda^{g}\right]\left(\mathcal{S}_{\text {glob }}\right.$ coincides with $\left.\mathcal{S}_{\text {elas }}\right)$. Consequently, neither approximation nor reduction are introduced with respect to the usual modal analysis, but only the displacements of the distinct structural levels are separated. In this case, we have $n_{g}=n=1,457$. Again, the methodology presented in Section 5 is applied using $\mathcal{A}_{\mathcal{L}}$ and $\mathcal{A}_{\mathcal{M}}$ with $N_{d}=5$ and $N_{d}=15$.

LF-scale basis. The calculated rank of the $\left(n_{g} \times n_{g}\right)$ real matrix $\left[M_{\mathcal{A}_{\mathcal{L}}}\right]$ is $\mathcal{N}_{\mathcal{L}}=$ 168. Similarly to the previous results, truncation order $n_{\mathcal{L}}$ is 168 , and the LF-scale basis is constituted of $n_{\mathcal{L}}=168 \mathrm{LF}$-scale modes $\left\{\boldsymbol{\varphi}_{\alpha}^{\mathcal{L}}\right\}_{\alpha}$.

MF-scale basis. The calculated rank of matrix $\left[M_{\mathcal{A}_{\mathcal{M}}}\right]$ is $\mathcal{N}_{\mathcal{M}}=1,457$ (which corresponds to the value of $n_{g}$ ). As in Section 6.2.1, truncation order $\nu_{\mathcal{M}}$ is 479 
and the MF-scale basis is constituted of $n_{\mathcal{M}}=\nu_{\mathcal{M}}-n_{\mathcal{L}}=311$ MF-scale modes $\left\{\boldsymbol{\varphi}_{\beta}^{\mathcal{M}}\right\}_{\beta}$.

$H F$-scale basis. Since $n_{\mathcal{H}}=n_{g}-n_{\mathcal{L}}-n_{\mathcal{M}}$, the HF-scale basis is constituted of $n_{\mathcal{H}}=978 \operatorname{modes}\left\{\varphi_{\gamma}^{\mathcal{H}}\right\}_{\gamma}$.

For each one of these three bases, the modal density is plotted in Fig. 15. Comparing with the previous results of Section 6.1, there are numerous HF-scale modes $\left\{\varphi_{\gamma}^{\mathcal{H}}\right\}_{\gamma}$ in the high part of the frequency band.

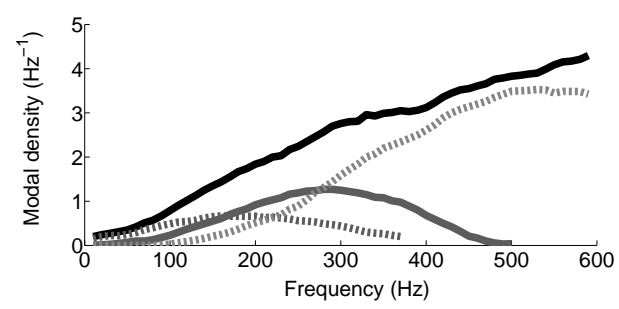

Figure 15: Modal densities: elastic modes $\left(n=1,457\right.$; black solid line), LF-scale modes ( $n_{\mathcal{L}}=$ 168 ; gray dashed line), MF-scale modes $\left(n_{\mathcal{M}}=311\right.$; gray solid line), and HF-scale modes $\left(n_{\mathcal{H}}=978\right.$; light-gray dashed line).

For each one of observation nodes obs1, obs2, obs3, and obs4, and for $\mathcal{I}$ equal to $\mathcal{L}, \mathcal{M}$, and $\mathcal{H}$, Fig. 16 displays the graphs of the FRFs related to $\mathbb{U}^{\mathcal{I}}(\omega)$. The conclusions are the same as for Section 6.1, except for the HF-scale basis that contributes more in the high part of the frequency band. Figure 17 displays the FRFs related to $\mathbb{U}^{\mathcal{L}}(\omega), \mathbb{U}^{\mathcal{L} \mathcal{M}}(\omega)$, and $\mathbb{U}^{\left(n_{\mathcal{L}}, n_{\mathcal{M}}, n_{\mathcal{H}}\right)}(\omega)$. The FRFs related to $\mathbb{U}^{\mathcal{L}}(\omega)$ and $\mathbb{U}^{\mathcal{L M}}(\omega)$ are close to the FRFs related to $\mathbb{U}^{\left(N_{d}, n_{g}\right)}(\omega)$ computed in Section 6.1 with Eq. (20) for which $N_{d}=5\left(n_{g}=168\right)$ and $N_{d}=15\left(n_{g}=479\right)$. On the other hand, the response $\mathbb{U}^{\left(n_{\mathcal{L}}, n_{\mathcal{M}}, n_{\mathcal{H}}\right)}(\omega)$ is, as expected, exactly superimposed to the reference. 

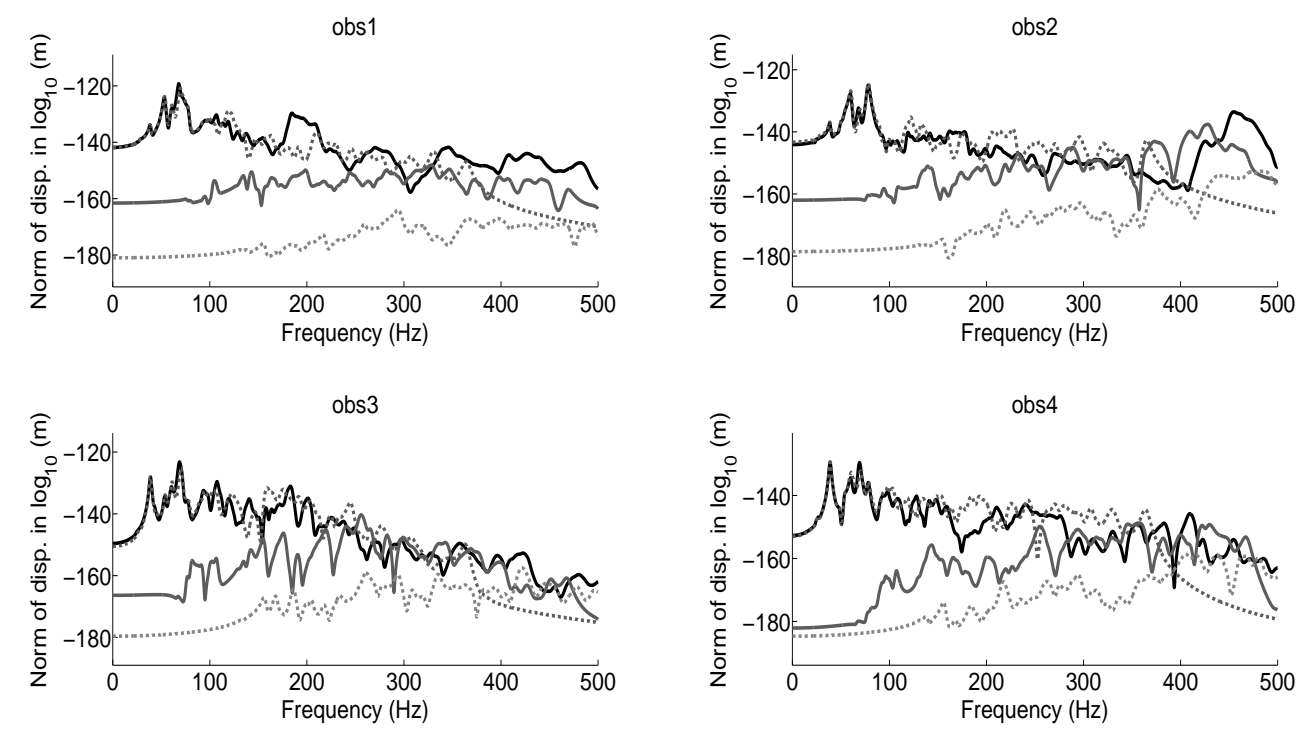

Figure 16: FRF of the norm of the displacements in log scale, for observation nodes obs1, obs2, obs3, and obs4, using: the elastic modes (black solid line), the LF-scale modes (gray dashed line), the MF-scale modes (gray solid line), and the HF-scale modes (light-gray dashed line).
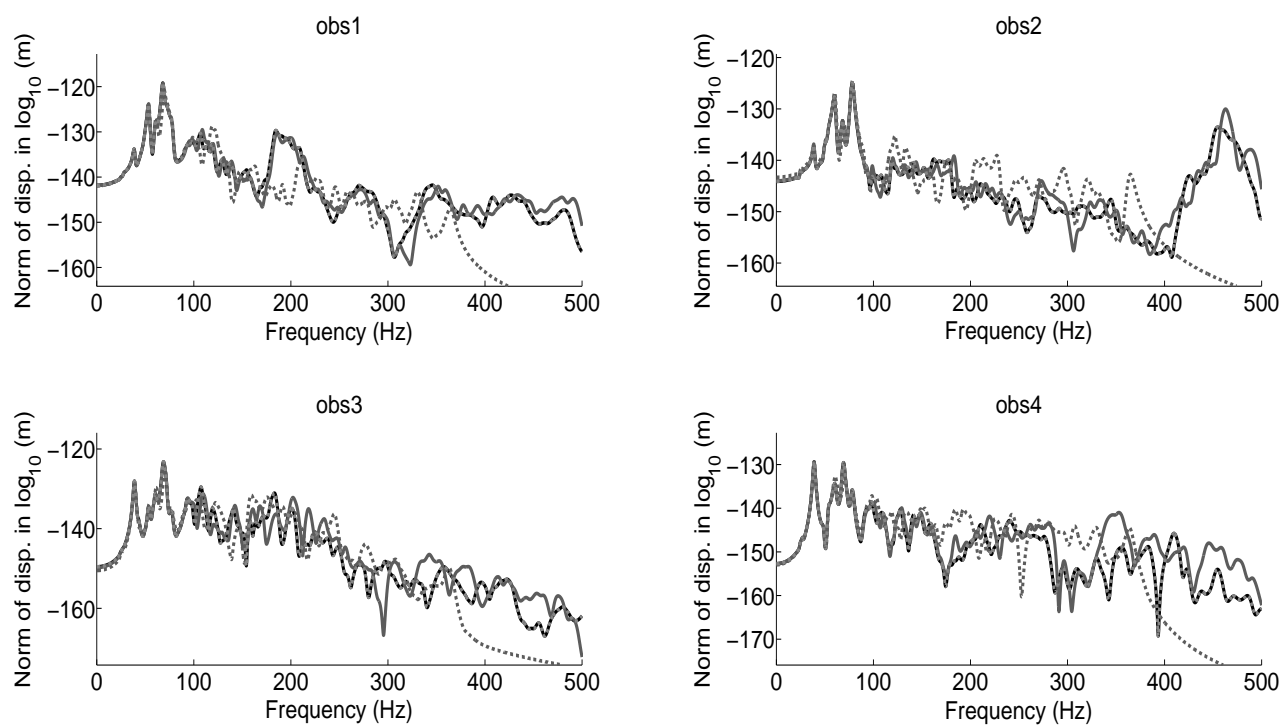

Figure 17: FRF of the norm of the displacements in log scale, for observation nodes obs1, obs2, obs3, and obs4, using: (1) the elastic modes (black solid line); (2) the LF-scale modes (gray dashed line); (3) the LF-scale modes and the MF-scale modes (gray solid line); (4) the LF-scale modes, the MF-scale modes, and the HF-scale modes (light-gray dashed line). 


\section{Conclusions}

A general method has been presented for constructing a small-dimension ROM of global displacements, which is adapted for analyzing the dynamical responses of the stiff master part of complex structures exhibiting numerous local elastic modes in the whole frequency band (including the LF band) due to the presence of numerous flexible parts and to the structural complexity. The dimension of the ROM constructed is smaller than the dimension of the classical ROM that would be constructed using the usual modal analysis. The method proposed has been especially developed to be non-intrusive with respect to the commercial softwares, and induces a negligible additional numerical cost with respect to the usual modal analysis. The method has been applied to the complex computational model of an automobile, for which the reduction factor of the ROM dimension is about 3. A detailed convergence analysis of the global-displacements ROM has been carried out and validates the proposed method dedicated to the construction of a smalldimension ROM whose accuracy is controlled.

Furthermore, a general method has been proposed for constructing a multilevel representation of the solution space, which is expressed as a direct sum of constructed subspaces (that are associated with the distinct structural levels). The purpose of the multilevel ROM is to allow the implementation of separated physical modelings depending on the family of modes. In this method, these families of modes are constructed in projecting the physical computational model onto the adjusted global-displacements subspace of reduced dimension, such that the computational cost allocated to their construction is negligible.

\section{Acknowledgements}

This research was supported by Agence Nationale de la Recherche, Contract HiMoDe, ANR-2011-BLAN-00378. The authors thank PSA Peugeot Citroën for the authorization to use the automobile computational model.

\section{References}

[1] Soize C. Medium frequency linear vibrations of anisotropic elastic structures. La Recherche Aérospatiale (English edition) 1982; 5:65-87.

[2] Ohayon R, Soize C. Advanced Computational Vibroacoustics - ReducedOrder Models and Uncertainty Quantification. New York: Cambridge University Press; 2014. 
[3] Bathe KJ, Wilson, EL. Numerical Methods in the Finite Element Method. Englewood Cliffs, NJ: Prentice-Hall; 1976.

[4] Meirovitch L. Dynamics and Control of Structures. New York: Wiley; 1990.

[5] Argyris J, Mlejnek HP. Dynamics of Structures. Amsterdam: North-Holland; 1991.

[6] Ohayon R, Soize C. Structural acoustics and vibration. London: Academic Press; 1998.

[7] Casciati S, Faravelli L. Quantity vs. quality in the model order reduction (MOR) of a linear system. Smart Structures and Systems 2014, 13(1): 99109.

[8] Lyon RH, DeJong RG. Theory and Application of Statistical Energy Analysis. Boston, MA: Butterworths-Heimann; 1995.

[9] Craig RR, Kurdila AJ. Fundamentals of Structural Dynamics. New Jersey: Wiley, 2nd Edition, John Wiley and Sons; 2006.

[10] Bampton MCC, Craig RR. Coupling of substructures for dynamic analyses. American Institute of Aeronautics and Astronautics Journal 1968; 6(7): 1313-1319.

[11] Mac Neal R. A hybrid method of component mode synthesis. Computers and Structures 1971, 1(4): 581-601.

[12] Rubin S. Improved component-mode representation for structural dynamic analysis. American Institue of Aeronautics and Astronautics Journal 1975; 13(8): 995-1006.

[13] Farhat C, Geradin M. On a Component Mode Synthesis Method and Its Application to Incompatible Substructures. Computers and Structures 1994; 51(5): 459-473.

[14] Soize C, Batou A. Stochastic reduced-order model in low-frequency dynamics in presence of numerous local elastic modes. Journal of applied mechanics - Transactions of the ASME 2011; 78(6): 061003. 
[15] Arnoux A, Batou A, Soize C, Gagliardini L. Stochastic reduced order computational model of structures having numerous local elastic modes in low frequency dynamics. Journal of Sound and Vibration 2013, 332(16): 36673680 .

[16] Arnoux A, Soize C, Batou A, Gagliardini L. Reduced-order computational model for low-frequency dynamics of automobiles. Advances in Mechanical Engineering 2013; Article ID 310362: 1-12.

[17] Bathe KJ. Finite element procedures. Upper Saddle River, New Jersey: Prentice Hall; 1996.

[18] Zienkiewicz OC, Taylor RL. The Finite Element Method. Oxford: Butterworth-Heinemann, 5th ed.; 2000.

[19] Sethian JA. A Fast Marching Level Set Method for Monotonically Advancing Fronts. Proceedings of the National Academy of Sciences 1996; 93(4): 1591-1595.

[20] Sethian JA, Kimmel R. Computing Geodesic Paths on Manifolds, Proceedings of the National Academy of Sciences 1998; 95: 8431-8435.

[21] Soize C, Desceliers C. Computational aspects for constructing realizations of polynomial chaos in high dimension. SIAM Journal On Scientific Computing 2010; 32(5): 2820-2841.

[22] Golub GH, Van Loan CF. Matrix Computations. Baltimore: John Hopkins Univ Press; 1983.

[23] Ezvan O, Batou A, Soize C. Reduced-order model for the dynamical analysis of complex structures with a high modal density. The 21st International Congress on Sound and Vibration (ICSV21) 2014; vol.2, ID 1396.

[24] Bathe KJ. The subspace iteration method - Revisited. Computers and Structures 2013; 126: 177-183.

[25] Durand JF, Soize C, Gagliardini L. Structural-acoustic modeling of automotive vehicles in presence of uncertainties and experimental identification and validation. Journal of the Acoustical Society of America 2008; 124(3): 15131525 . 
[26] Gagliardini L. Dispersed vibroacoustic responses of industrial products: what are we able to predict?. Proceedings of ISMA2014 including USD2014 2014; KEY session.

[27] Soize C. A nonparametric model of random uncertainties for reduced matrix models in structural dynamics. Probabilistic Engineering Mechanics 2000; 15(3): 277-294. 